\title{
VORTEX DYNAMICS FOR THE GINZBURG-LANDAU EQUATION WITH NEUMANN CONDITION
}

\author{
SHUICHI JIMBO* AND YOSHIHISA MORITA ${ }^{\dagger}$
}

\begin{abstract}
We study the Ginzburg-Landau equation, $u_{t}=\Delta u+\left(1 / \epsilon^{2}\right)\left(1-|u|^{2}\right) u, u=\left(u_{1}, u_{2}\right)$, in a planar contractible domain with Neumann boundary condition, where $\epsilon>0$ is a small parameter. We construct a finite-dimensional manifold of a family of approximate solutions and consider the dynamics near the manifold. If a solution lies in a sufficiently small neighborhood of the manifold, we can derive the dynamics projected on the manifold. Then the equation describing this dynamics provides a motion law of zeros to the approximate solutions and it approximates the dynamics of zeros (called vortices) of the original solution.
\end{abstract}

1. Introduction. We devote to the Ginzburg-Landau equation in a bounded domain $\Omega$ of $\mathbb{R}^{2}$ with the Neumann boundary condition:

$$
\begin{aligned}
& u_{t}=\Delta u+\frac{1}{\epsilon^{2}}\left(1-|u|^{2}\right) u=0, \quad(x, t) \in \Omega \times(0, \infty), \\
& \frac{\partial u}{\partial \nu}=0, \quad(x, t) \in \partial \Omega \times(0, \infty),
\end{aligned}
$$

where $u=\left(u_{1}(x, t), u_{2}(x, t)\right)^{T}, \epsilon$ is a small positive parameter and $\partial / \partial \nu$ denotes the outer normal derivative on the smooth boundary $\partial \Omega$. This equation is a gradient equation of the following energy functional

$$
E_{\epsilon}(u):=\frac{1}{2} \int_{\Omega}\left\{|\nabla u|^{2}+\frac{1}{2 \epsilon^{2}}\left(1-|u|^{2}\right)^{2}\right\} d x
$$

in $H^{1}\left(\Omega ; \mathbb{R}^{2}\right)$. Moreover the Euler equation of $E_{\epsilon}(u)$ is a system of elliptic equations with the Neumann boundary condition

$$
\begin{aligned}
& \Delta u+\frac{1}{\epsilon^{2}}\left(1-|u|^{2}\right) u=0, \quad x \in \Omega, \\
& \frac{\partial u}{\partial \nu}=0, \quad x \in \partial \Omega
\end{aligned}
$$

whose solution gives an equilibrium state of (1.1). Throughout the paper we assume that the domain $\Omega$ is contractible. We also allow the complex expression $u=u_{1}(x, t)+$ $i u_{2}(x, t)$ for a solution $u=\left(u_{1}(x, t), u_{2}(x, t)\right)^{T}$ to $(1.1)$.

Since a solution $u(x, t)$ of (1.1) has two components, a vector field on $\Omega$ can be defined by the solution for each time $t>0$ and the zero set of the solution at time $t$ is consist of discrete points in generic. Note that the degree of each zero $y$ can be defined by $\operatorname{deg}\left(y, \partial B_{\rho}(y)\right)$, where $B_{\rho}(y)$ is a disk with a small radius $\rho$. Zeros of the equation (1.1) (or (1.4)) are called vortices which play an important role for characterizing some dynamical behavior of the solution (refer to the introduction of

\footnotetext{
*Department of Mathematics, Hokkaido University, Sapporo 060-0810, Japan (jimbo@math.hokudai.ac.jp).

†Department of Applied Mathematics and Informatics, Faculty of Science and Technology, Ryukoku University, Seta Otsu 520-2194, Japan (morita@rins.ryukoku.ac.jp).
} 
[16]). Therefore the dynamics of vortices has been extensively studied by physicists and mathematicians, for instance see [6], [12], [19], [20], [21], [22], [23], [26].

Among other things, as for the equation (1.1) with the Dirichlet boundary data

$$
u=g(x), \quad x \in \partial \Omega, \quad|g|=1, \quad \operatorname{deg}(g ; \partial \Omega)=d, \quad d \in \mathbb{Z} \backslash\{0\}
$$

(hereafter we assume $d>0$ for simplicity of notation), elaborate results were proved by Lin $([19,20])$ and Jerrard-Soner $([12])$. They derived, taking a limit of a subsequence $\epsilon_{n} \rightarrow 0$ after time scaling $s=t / \log (1 / \epsilon)$, a limit equation describing the motion law of the vortices. If we let $\left(a_{1}, \cdots, a_{d}\right)$ be a configuration of the vortices, the limit equation is written as

$$
\frac{d}{d s}\left(a_{1}, \cdots, a_{d}\right)=-\frac{1}{\pi} \operatorname{grad} W\left(a_{1}, \ldots, a_{d}\right),
$$

where $W\left(\boldsymbol{a}_{1}, \ldots, \boldsymbol{a}_{d}\right)$ is the renormalized energy given by [2]. (Note that RubinsteinSternberg [26] first showed rigorously that the scaling $s=t / \log (1 / \epsilon)$ works in the study of vortex dynamics.)

On the other hand for the Neumann problem, (1.1)-(1.2), Lin [21] derived the limit equation as done for the Dirichlet case by defining the renormalized energy appropriately. Although the renormalized energy is not explicitly given there (except for the special case, $\Omega=\{|x|<1\}$ and $\mathrm{d}=1$ ), it suggests important dynamical properties of vortices, for example, the annihilation of two vortices with opposite signs of degree.

To investigate the dynamics of the limit equation extensively, we need more information on the limit equation. A recent study in [16] provides an explicit form of the limit equation for the Neumann problem. In fact, after deriving the limit equation by applying the argument of [12] to the Neumann case, we succeeded to rewrite it with a Green function and Robin function of it. We present it here. Let the configuration of $m$-vortices be denoted by

$$
\left(y^{(1)}(s), \cdots, y^{(m)}(s)\right) \in \hat{\Omega}^{m}:=\Omega \times \cdots \times \Omega .
$$

Then the limit equation can be written as follows:

$$
\frac{d}{d s} y^{(j)}=\nabla S\left(y^{(j)}\right)+2 \sum_{k \neq j}^{m} d_{j} d_{k} \nabla_{x} G\left(y^{(j)}, y^{(k)}\right) \quad(j=1, \ldots, m)
$$

where time $s$ is scaled as in $(1.7), d_{j}(=1$ or -1$)$ denotes degree of the vortex $y^{(j)}$, $G(x, y)$ is the Green function of $\Delta$ with Dirichlet condition and $S(x)$ is the Robin function of it. With the aid of this form some dynamical properties (existence or nonexistence of equilibria, collision of vortices etc.) of the limit equation are also shown in [16].

In this paper we study the dynamics of solutions to (1.1)-(1.2) for sufficiently small but positive $\epsilon$ and show that the motion law of the vortices can be approximated by the limit equation under an appropriate condition. In fact given positive $\rho_{0}>0$, we construct a family of approximate solutions

$$
\begin{aligned}
& u=u_{\epsilon}(x ; \boldsymbol{y}), \quad \boldsymbol{y}=\left(y^{(1)}, \cdots, y^{(m)}\right) \in Y, \\
& Y:=\left\{\boldsymbol{y} \in \hat{\Omega}^{m}:\left|y^{(j)}-y^{(k)}\right| \geq 2 \rho_{0}, j \neq k, \quad \operatorname{dist}\left(y^{(j)}, \partial \Omega\right) \geq \frac{3}{2} \rho_{0}\right\} .
\end{aligned}
$$

Then we obtain a $2 m$-dimensional submanifold

$$
\mathcal{M}_{\epsilon}:=\{u=u(\cdot ; \boldsymbol{y}): \boldsymbol{y} \in Y\}
$$


in the space of continuous functions $C^{0}\left(\bar{\Omega} ; \mathbb{R}^{2}\right)$. We will prove that for a positive function $\delta(\epsilon)=o(\epsilon)$, there is a $C^{1}$ function $\boldsymbol{y}_{\epsilon}(\cdot)$ from the neighborhood of $\mathcal{M}_{\epsilon}$

$$
\mathcal{U}\left(\mathcal{M}_{\epsilon}\right)=\left\{u \in C^{0}\left(\bar{\Omega} ; \mathbb{R}^{2}\right):\left\|u-u_{\epsilon}(\cdot ; \boldsymbol{y})\right\|_{C^{0}(\bar{\Omega})}<\delta(\epsilon), \boldsymbol{y} \in Y\right\}
$$

into $Y$ such that for a solution $u(x, t) \in \mathcal{U}\left(\mathcal{M}_{\epsilon}\right), \boldsymbol{y}(t)=\boldsymbol{y}_{\epsilon}(u(\cdot, t))$ satisfies

$$
\begin{aligned}
& \log (1 / \epsilon) \frac{d}{d t} y^{(j)}=-\frac{1}{\pi} \frac{\partial}{\partial y^{(j)}} V(\boldsymbol{y})+o(1) \\
& V(\boldsymbol{y}):=-\pi \sum_{k=1}^{m} S\left(y^{(k)}\right)-\pi \sum_{k=1}^{m} \sum_{j \neq k} G\left(y^{(j)}, y^{(k)}\right) .
\end{aligned}
$$

Hence by scaling $s=t / \log (1 / \epsilon)$, the leading term of this equation agrees with (1.8). To obtain the above result, we need to verify

$$
\begin{aligned}
& E_{\epsilon}\left(u_{\epsilon}(\cdot, \boldsymbol{y})\right)=-\pi \sum_{k=1}^{m} S\left(y^{(k)}\right)-\pi \sum_{k=1}^{m} \sum_{j \neq k} G\left(y^{(j)}, y^{(k)}\right)+o(1) \\
& \frac{\partial}{\partial y^{(j)}} E_{\epsilon}\left(u_{\epsilon}(\cdot, \boldsymbol{y})\right)=-\frac{\partial}{\partial y^{(j)}} V(\boldsymbol{y})+o(1) .
\end{aligned}
$$

In fact once we obtained these relations, we can derive the above motion law near the manifold by applying the idea found in [7].

We note that one can find many papers on the dynamics of interfaces and spikes for a class of reaction-diffusion equations including Allen-Cahn equation and GiererMeinhardt equation (for instance see [1], [4], [9], [8], [10], and the references therein). It is also interesting that a similar form of the energy (1.12) is addressed in [27], where it is shown that a critical point of the energy gives a locus of spikes to a spike solution. In many cases of the reaction-diffusion equations it can be proved that normal direction of manifolds for approximate solutions possesses the attractivity; thus the dynamics near the manifold is controlled by that on the manifold. Such attractivity could be proved by solving eigenvalue problem of the linearized operator at the approximate solutions or the comparison method for the scalar equation.

On the other hand for the Ginzburg-Landau equation it is not so easy to handle the eigenvalue problem of the linearized operator for vortex solutions. Actually we only have a few results for it (see [15], [23], [24]) and we can't apply them to the present case. Therefore we need to study further to prove that the solution remain in a neighborhood of the manifold for a long time. Although the study developed in this article is not complete for this reason, we believe that it would work in the future study.

This paper is organized as follows. In $\S 2$ given prescribed distinct points $y^{(j)}, 1 \leq$ $j \leq m$, we consider a harmonic map from $\Omega \backslash\left\{y^{(1)}, \cdots, y^{(m)}\right\}$ into $S^{1} \subset \mathbb{C}$ with Neumann condition. This map has singularity at each $y^{(j)}$. We compute the energy of the map in a domain with holes around the points $y^{(j)}, 1 \leq j \leq m$. On the other hand in $\S 3$ we compute the energy of the symmetric vortex solution in a small disk centered at the zero of the solution. In $\S 4$ and $\S 5$ we investigate an approximate solution parametrized by $\left\{y^{(1)}, \cdots, y^{(m)}\right\}$, which denote the locus of zeros, and obtain (1.12) and (1.13). By combination of those results we derive (1.10)-(1.11) describing the dynamics near the manifold of the approximate solution in $\S 6$. 
2. Energy of the harmonic map with singularity. Given $p \in \Omega$, let $\varphi(x ; p)$ be a solution of

$$
\begin{cases}\Delta_{x} \varphi=0, & x \in \Omega, \\ \frac{\partial \varphi}{\partial \nu_{x}}=-\left\langle\nu_{x}, \nabla_{x} \operatorname{Arg}(x-p)\right\rangle, & x \in \partial \Omega\end{cases}
$$

where $\langle\cdot, \cdot\rangle$ denotes the inner product of vectors in $\mathbb{R}^{2}$. Although $\operatorname{Arg}(x-p)$ is multivalued function with a singularity at $x=p$, each representative satisfies

$$
\nabla_{x} \operatorname{Arg}(x-p)=\frac{(x-p)^{\perp}}{|x-p|^{2}}, \quad(x-p)^{\perp}:=\left(-\left(x_{2}-p_{2}\right),\left(x_{1}-p_{1}\right)\right) .
$$

In fact $\nabla_{x} \operatorname{Arg}(x-p)$ is well-defined as a single-valued vector function (see [17]). By the condition

$$
\int_{\partial \Omega}\left\langle\nu_{x}, \nabla_{x} \operatorname{Arg}(x-p)\right\rangle d S=0
$$

(2.1) has a solution which is unique up to additive constants (see [16]).

We denote a disk with radius $\rho$ centered at $x=\boldsymbol{a}$ by

$$
B_{\rho}(a):=\{|x-a|<\rho\}
$$

and denote the degree of a function $u=\left(u_{1}(x), u_{2}(x)\right)^{T}$ around $x=\boldsymbol{a}$ by $\operatorname{deg}\left(u(\cdot) ; \partial B_{\rho}(\boldsymbol{a})\right)$. Let

$$
\hat{\Omega}^{m}:=\left\{\left(y^{(1)}, \ldots, y^{(m)}\right) \in R^{2 m}: y^{(j)} \in \Omega \quad(1 \leq j \leq m), y^{(j)} \neq y^{(k)} \quad(j \neq k)\right\} .
$$

Given $\boldsymbol{y}=\left(y^{(1)}, \ldots, y^{(m)}\right) \in \hat{\Omega}^{m}$, define

$$
\Theta(x ; \boldsymbol{y}):=\sum_{j=1}^{m} d_{j}\left(\operatorname{Arg}\left(x-y^{(j)}\right)+\varphi\left(x ; y^{(j)}\right)\right)
$$

and

$$
u_{h}(x ; \boldsymbol{y})=\exp (i \Theta(x ; \boldsymbol{y}))
$$

where $d_{j}=1$ or -1 . Note that $d_{j}=\operatorname{deg}\left(u_{h}(x ; \boldsymbol{y}), \partial B_{\rho}\left(y^{(j)}\right)\right)$. We easily check that

(2.7) $\operatorname{div}(\nabla \Theta)=0, \quad x \in \Omega, x \neq y^{(j)}(1 \leq j \leq m), \quad \frac{\partial \Theta}{\partial \nu}=0, \quad x \in \partial \Omega$,

$$
\Delta u_{h}+\left|\nabla u_{h}\right|^{2} u_{h}=0, \quad x \in \Omega, x \neq y^{(j)}(1 \leq j \leq m), \quad \frac{\partial u_{h}}{\partial \nu}=0, \quad x \in \partial \Omega
$$

Hence $u_{h}$ is a harmonic map from $\Omega \backslash\left\{y^{(1)}, \cdots, y^{(m)}\right\}$ into $S^{1} \subset \mathbb{C}$, having singularity at $x=y^{(j)}, j=1, \ldots, m$.

We introduce the conjugate harmonic function $S(x, p)$ to $\varphi(x ; p)$. The CauchyRiemann equation implies

$$
\nabla_{x} S(x, p)^{\perp}=\nabla_{x} \varphi(x ; p)
$$


Then a solution to the elliptic equation

$$
\Delta_{x} S=0, \quad x \in \Omega, \quad S=-\log |x-p|, \quad x \in \partial \Omega
$$

gives the conjugate harmonic function. Indeed it follows from the facts that the domain is contractible and that the solution $S=S(x, p)$ to $(2.10)$ admits the boundary condition

$$
\frac{\partial}{\partial \nu_{x}} S^{\perp}=-\left\langle\tau, \nabla_{x} S\right\rangle=\frac{\langle\tau,(x-p)\rangle}{|x-p|^{2}}=-\frac{\left\langle\nu,(x-p)^{\perp}\right\rangle}{|x-p|^{2}}=\frac{\partial}{\partial \nu_{x}} \varphi,
$$

where $\tau$ denotes the unit tangential vector on $\partial \Omega$.

Set

$$
H(x, p):=\operatorname{Arg}(x-p)+\varphi(x ; p) .
$$

Then we have the following lemma:

LEMma 2.1. Given $p \in \Omega$, define

$$
G(x, p):=\log |x-p|+S(x, p), \quad x \neq p, x \in \Omega .
$$

Then

$$
\nabla_{x} G(x, p)^{\perp}=\nabla_{x} H(x, p), \quad x \neq p, x \in \Omega,
$$

and with an appropriate additive constant to $G(x, p)$,

$$
\begin{cases}\Delta_{x} G=0, & x \in \Omega \backslash\{p\}, \\ G=0, & x \in \partial \Omega, \\ G(x, p) \sim \log |x-p|+O(1), & x \approx p, x \neq p\end{cases}
$$

hold, namely $G(x, p)$ is the Green function of the Laplacian $\Delta$ with Dirichlet (zero) boundary condition.

The next proposition is the main result of the present section.

Proposition 2.2. Let $\rho$ be a positive number satisfying

$$
\rho<\min \left\{\frac{1}{2} \min _{1 \leq j \leq m} \operatorname{dist}\left(\partial \Omega, y^{(j)}\right), \quad \frac{1}{3} \min _{1 \leq j, k \leq m, j \neq k}\left|y^{(j)}-y^{(k)}\right|\right\} .
$$

Set $\Omega_{\rho}:=\Omega \backslash\left(\cup_{i=1}^{m} \overline{B_{\rho}\left(y^{(j)}\right)}\right)$ and

$$
E_{\rho}\left(u_{h}\right):=\frac{1}{2} \int_{\Omega_{\rho}}\left|\nabla_{x} u_{h}(x ; \boldsymbol{y})\right|^{2} d x
$$

Then

$$
E_{\rho}\left(u_{h}\right)=-m \pi \log \rho+V(\boldsymbol{y})+O(\rho)
$$

where

$$
V(\boldsymbol{y}):=-\pi \sum_{j=1}^{m} S\left(y^{(j)}, y^{(j)}\right)-\pi \sum_{k=1}^{m} \sum_{j \neq k} d_{j} d_{k} G\left(y^{(j)}, y^{(k)}\right)
$$

To prove this proposition we prepare two lemmas.

LEMMA 2.3. 
(i) $E_{\rho}\left(u_{h}\right)$ in $(2.15)$ satisfies

$$
\begin{aligned}
E_{\rho}\left(u_{h}\right)= & -\frac{1}{2} \sum_{k=1}^{m}\left\{\int_{\partial B_{\rho}\left(y^{(k)}\right)} G\left(x, y^{(k)}\right) \frac{\partial}{\partial \nu_{x}} G\left(x, y^{(k)}\right) d \sigma\right. \\
& \left.+\sum_{j, \ell}^{\prime} d_{j} d_{\ell} \int_{\partial B_{\rho}\left(y^{(k)}\right)} G\left(x, y^{(j)}\right) \frac{\partial}{\partial \nu_{x}} G\left(x, y^{(\ell)}\right) d \sigma\right\},
\end{aligned}
$$

where $\sum_{j, \ell}^{\prime}$ denotes the summation over indices, $1 \leq j, \ell \leq m, j \neq k$ or $\ell \neq k$.

(ii) Define

$$
F_{\rho}^{(k)}:=\frac{1}{2} \int_{B_{\rho}\left(y^{(k)}\right)}\left(\left|\nabla u_{h}\right|^{2}-\frac{1}{\left|x-y^{(k)}\right|^{2}}\right) d x
$$

Then

$$
\begin{aligned}
& \text { (2.20) } F_{\rho}^{(k)}=-\pi S\left(y^{(k)}, y^{(k)}\right)-\pi \sum_{j \neq k} d_{k} d_{j} G\left(y^{(j)}, y^{(k)}\right) \\
& +\frac{1}{2}\left\{\int_{\partial B_{\rho}\left(y^{(k)}\right)} S\left(x, y^{(k)}\right) \frac{\partial}{\partial \nu_{x}} \log \left|x-y^{(k)}\right| d \sigma+G\left(x, y^{(k)}\right) \frac{\partial}{\partial \nu_{x}} S\left(x, y^{(k)}\right) d \sigma\right\}
\end{aligned}
$$$$
+\sum_{j, \ell}^{\prime} d_{j} d_{\ell} \int_{\partial B_{\rho}\left(y^{(k)}\right)} G\left(x, y^{(j)}\right) \frac{\partial}{\partial \nu_{x}} G\left(x, y^{(\ell)}\right) d \sigma
$$

where $\sum_{j, k}^{\prime}$ is as in (i).

\section{LEMMA 2.4. The identity}

$$
E_{\rho}\left(u_{h}\right)+\sum_{k=1}^{m} F_{\rho}^{(k)}=-m \pi \log \rho+V(\boldsymbol{y})
$$

holds.

Before proving Lemmas 2.3, 2.4, we prove Proposition 2.2 with the aid of Lemma 2.4. Proof of Proposition 2.2: We see that there is a positive constant such that

$$
\left.|| \nabla u_{h}\right|^{2}-\frac{1}{\left|x-y^{(k)}\right|^{2}} \mid \leq \frac{C_{1}}{\left|x-y^{(k)}\right|}, \quad x \in B_{\rho}\left(y^{(k)}\right),
$$

where $C_{1}$ is independent of $\rho$. Therefore we obtain

$$
\left.\int_{B_{\rho}\left(y^{(k)}\right)}|| \nabla u_{h}\right|^{2}-\frac{1}{\left|x-y^{(k)}\right|^{2}} \mid \leq 2 \pi C_{1} \int_{0}^{\rho} \frac{1}{r} r d r=2 \pi C_{1} \rho
$$

from which

$$
\left|F_{\rho}^{(k)}\right| \leq C \rho
$$

follows. Hence we obtain the assertion of the proposition by Lemma 2.4. 
Now we prove Lemmas 2.3 and 2.4 in the rest of this section. We first prove Lemma 2.4.

Proof of Lemma 2.4 : We can verify

$$
\begin{aligned}
& \int_{\partial B_{\rho}\left(y^{(k)}\right)} G\left(x, y^{(k)}\right) \frac{\partial}{\partial \nu_{x}} G\left(x, y^{(k)}\right) d \sigma \\
= & \int_{\partial B_{\rho}\left(y^{(k)}\right)}\left(\log \left|x-y^{(k)}\right| \frac{\partial}{\partial \nu_{x}} \log \left|x-y^{(k)}\right|+S\left(x, y^{(k)}\right) \frac{\partial}{\partial \nu_{x}} \log \left|x-y^{(k)}\right|\right) d \sigma \\
& \quad \int_{\partial B_{\rho}\left(y^{(k)}\right)} G\left(x, y^{(k)}\right) \frac{\partial}{\partial \nu_{x}} S\left(x, y^{(k)}\right) d \sigma \\
= & 2 \pi \log \rho+\int_{\partial B_{\rho}\left(y^{(k)}\right)} S\left(x, y^{(k)}\right) \frac{\partial}{\partial \nu_{x}} \log \left|x-y^{(k)}\right| d \sigma \\
& \quad+\int_{\partial B_{\rho}\left(y^{(k)}\right)} G\left(x, y^{(k)}\right) \frac{\partial}{\partial \nu_{x}} S\left(x, y^{(k)}\right) d \sigma .
\end{aligned}
$$

Here we computed

$$
\int_{\partial B_{\rho}\left(y^{(k)}\right)} \log \left|x-y^{(k)}\right| \frac{\partial}{\partial \nu_{x}} \log \left|x-y^{(k)}\right| d \sigma=\int_{0}^{2 \pi} \frac{\log \rho}{\rho} \rho d \theta=2 \pi \log \rho .
$$

Using (2.18),(2.19) and the above indentity, we obtain (2.21).

Proof of Lemma 2.3: We first prove (2.18). Put

$$
\psi^{(j)}:=d_{j}\left(\operatorname{Arg}\left(x-y^{(j)}\right)+\varphi\left(x, y^{(j)}\right)\right) .
$$

Then

$$
E_{\rho}\left(u_{h}\right)=\frac{1}{2} \int_{\Omega_{\rho}}\left|\sum_{j=1}^{m} \nabla_{x} \psi^{(j)}\right|^{2} d x=\frac{1}{2} \int_{\Omega_{\rho}} \sum_{j=1}^{m} \sum_{\ell=1}^{m} \nabla_{x} \psi^{(j)} \cdot \nabla_{x} \psi^{(\ell)} d x
$$

By Lemma 2.1 we have

$$
\nabla_{x} \psi^{(j)} \cdot \nabla_{x} \psi^{(\ell)}=d_{j} d_{\ell} \nabla_{x} G\left(x, y^{(j)}\right) \cdot \nabla_{x} G\left(x, y^{(\ell)}\right)
$$

therefore

$$
\begin{aligned}
E_{\rho}\left(u_{h}\right) & =\frac{1}{2} \sum_{j=1}^{m} \sum_{\ell=1}^{m} d_{j} d_{\ell} \int_{\Omega_{\rho}} \nabla_{x} G\left(x, y^{(j)}\right) \cdot \nabla_{x} G\left(x, y^{(\ell)}\right) d x \\
& =\frac{1}{2} \sum_{j=1}^{m} \sum_{\ell=1}^{m} d_{j} d_{\ell}\left(-\sum_{k=1}^{m} \int_{\partial B_{\rho}\left(y^{(k)}\right)} G\left(x, y^{(j)}\right) \frac{\partial}{\partial \nu_{x}} G\left(x, y^{(\ell)}\right) d \sigma\right) \\
& =-\frac{1}{2} \sum_{k=1}^{m} \sum_{j=1}^{m} \sum_{\ell=1}^{m} \int_{\partial B_{\rho}\left(y^{(k)}\right)} G\left(x, y^{(j)}\right) \frac{\partial}{\partial \nu_{x}} G\left(x, y^{(\ell)}\right) d \sigma
\end{aligned}
$$

which agrees with (2.18). 
Next we prove (2.20). We put

$$
\left\{\begin{array}{l}
F_{\rho}^{(k)}=J_{1}^{(k)}+J_{2}^{(k)} \\
J_{1}^{(k)}:=\frac{1}{2} \int_{B_{\rho}\left(y^{(k)}\right)}\left\{2 \nabla_{x} \operatorname{Arg}\left(x-y^{(k)}\right) \cdot \nabla \varphi\left(x ; y^{(k)}\right)+\left|\nabla_{x} \varphi\left(x ; y^{(k)}\right)\right|^{2}\right\} d x \\
J_{2}^{(k)}:=\frac{1}{2} \int_{B_{\rho}\left(y^{(k)}\right)}\left(\left|\nabla_{x} \Theta\right|^{2}-\left|\nabla_{x} \psi^{(k)}\right|^{2}\right) d x
\end{array}\right.
$$

In terms of (2.2) and (2.9) we can write

$$
\begin{aligned}
J_{1}^{(k)} & =\frac{1}{2} \int_{B_{\rho}\left(y^{(k)}\right)}\left\{2 \nabla_{x} \log \left|x-y^{(k)}\right| \cdot \nabla_{x} S\left(x, y^{(k)}\right)+\left|\nabla_{x} S\left(x, y^{(k)}\right)\right|^{2}\right\} d x \\
& =\frac{1}{2} \int_{B_{\rho}\left(y^{(k)}\right)}\left\{\nabla_{x} \log \left|x-y^{(k)}\right| \cdot \nabla_{x} S\left(x, y^{(k)}\right)+\nabla_{x} G\left(x, y^{(k)}\right) \cdot \nabla_{x} S\left(x, y^{(k)}\right)\right\} d x .
\end{aligned}
$$

We easily check

$$
\begin{aligned}
J_{1}^{(k)}=-\pi S\left(y^{(k)}, y^{(k)}\right) & +\frac{1}{2}\left\{\int_{\partial B_{\rho}\left(y^{(k)}\right)} S\left(x, y^{(k)}\right) \frac{\partial}{\partial \nu_{x}} \log \left|x-y^{(k)}\right| d \sigma\right. \\
& \left.+\int_{\partial B_{\rho}\left(y^{(k)}\right)} G\left(x, y^{(k)}\right) \frac{\partial}{\partial \nu_{x}} S\left(x, y^{(k)}\right) d \sigma\right\} .
\end{aligned}
$$

Next we compute $J_{2}^{(k)}$. Let

$$
\Psi^{(k)}:=\sum_{j \neq k} \psi^{(j)} .
$$

Then

$$
\begin{aligned}
J_{2}^{(k)}= & \frac{1}{2} \int_{B_{\rho}\left(y^{(k)}\right)}\left(2 \nabla_{x} \psi^{(k)} \cdot \nabla_{x} \Psi^{(k)}+\left|\nabla_{x} \Psi^{(k)}\right|^{2}\right) d x \\
= & \frac{1}{2} \int_{B_{\rho}\left(y^{(k)}\right)} \sum_{j \neq k} 2 d_{k} d_{j} \nabla_{x} G\left(x, y^{(k)}\right) \cdot \nabla_{x} G\left(x, y^{(j)}\right) d x \\
& +\frac{1}{2} \int_{B_{\rho}\left(y^{(k)}\right)} \sum_{j \neq k} \sum_{\ell \neq k} d_{j} d_{\ell} \nabla_{x} G\left(x, y^{(j)}\right) \cdot \nabla_{x} G\left(x, y^{(\ell)}\right) d x
\end{aligned}
$$

Note that

$$
\begin{aligned}
& \int_{B_{\rho}\left(y^{(k)}\right)} \nabla_{x} G\left(x, y^{(k)}\right) \cdot \nabla_{x} G\left(x, y^{(j)}\right) d x \\
= & -2 \pi G\left(y^{(k)}, y^{(j)}\right)+\int_{\partial B_{\rho}\left(y^{(k)}\right)} \frac{\partial}{\partial \nu_{x}} G\left(x, y^{(k)}\right) G\left(x, y^{(j)}\right) d \sigma
\end{aligned}
$$

$(2.27) \int_{B_{\rho}\left(y^{(k)}\right)} \nabla_{x} G\left(x, y^{(k)}\right) \cdot \nabla_{x} G\left(x, y^{(j)}\right) d x=\int_{\partial B_{\rho}\left(y^{(k)}\right)} G\left(x, y^{(k)}\right) \frac{\partial}{\partial \nu_{x}} G\left(x, y^{(j)}\right) d \sigma$ 
and for $j, \ell \neq k$,

$$
\int_{B_{\rho}\left(y^{(k)}\right)} \nabla_{x} G\left(x, y^{(j)}\right) \cdot \nabla_{x} G\left(x, y^{(\ell)}\right) d x=\int_{\partial B_{\rho}\left(y^{(k)}\right)} G\left(x, y^{(j)}\right) \frac{\partial}{\partial \nu_{x}} G\left(x, y^{(\ell)}\right) d \sigma
$$

holds. Applying (2.26), (2.27) and (2.28) to (2.25) yields

$$
\begin{aligned}
J_{2}^{(k)}=-\pi \sum_{j \neq k} d_{k} & d_{j} G\left(y^{(j)}, y^{(k)}\right) \\
& +\frac{1}{2} \sum_{j, \ell}^{\prime} d_{j} d_{\ell} \int_{\partial B_{\rho}\left(y^{(k)}\right)} G\left(x, y^{(j)}\right) \frac{\partial}{\partial \nu_{x}} G\left(x, y^{(\ell)}\right) d \sigma
\end{aligned}
$$

Adding (2.24) to (2.29), we obtain (2.20).

REMARK 2.5. Recall that the function $S(x):=S(x, x)$ is called a Robin function. Hence in the expressions of (2.17) and (2.20) we can use this Robin function.

3. Energy of the symmetric vortex solution. We let $f_{\infty}(s)$ be a unique solution to

$$
\left\{\begin{array}{l}
\frac{d^{2}}{d s^{2}} f+\frac{1}{s} \frac{d}{d s} f-\frac{1}{s^{2}} f+\left(1-f^{2}\right) f=0, \quad 0<s<\infty \\
f(0)=0, \quad f(\infty)=1, \quad 0<f(s)<1 \quad(0<s<\infty)
\end{array}\right.
$$

It has the asymptotics such that for a sufficiently large number $R_{1}>0$,

$$
\left\{\begin{array}{l}
f_{\infty}(s)=1-\frac{1}{2 s^{2}}+O\left(1 / s^{4}\right), \quad s>R_{1} \\
f_{\infty}(s)=a_{1} s+O\left(s^{3}\right), \quad s<1 / R_{1}
\end{array}\right.
$$

where $a_{1}$ is a positive constant (see [5] or [18]). Define

$$
f_{\epsilon}(r):=f_{\infty}(r / \epsilon)
$$

Then $\tilde{u}_{\epsilon}=f_{\epsilon}(r) e^{i \theta}$ gives a symmetric vortex solution to

$$
\Delta u+\frac{1}{\epsilon^{2}}\left(1-|u|^{2}\right) u=0, \quad x \in \mathbb{R}^{2} .
$$

Note that by (3.2)

$$
\begin{cases}f_{\epsilon}(r)=1-\frac{\epsilon^{2}}{2 r^{2}}+O\left(\epsilon^{4} / r^{4}\right), & \\ f_{\epsilon}^{\prime}(r)=\frac{\epsilon^{2}}{r^{3}}+O\left(\epsilon^{4} / r^{5}\right), & r>\epsilon R_{1} \\ f_{\epsilon}^{\prime \prime}(r)=\frac{\epsilon^{2}}{r^{4}}+O\left(\epsilon^{4} / r^{6}\right) & \end{cases}
$$


$\left({ }^{\prime}=d / d r,{ }^{\prime \prime}=d^{2} / d r^{2}\right)$. Given $\rho>0$, define

$$
I_{\epsilon}(\rho):=\frac{1}{2} \int_{B_{\rho}(0)}\left\{\left|\nabla \tilde{u}_{\epsilon}\right|^{2}+\frac{1}{2}\left(1-\left|\tilde{u}_{\epsilon}\right|^{2}\right)^{2}\right\} d x .
$$

Then we easily verify

$$
I_{\epsilon}(\rho)=\pi \int_{0}^{\rho}\left\{\left|f_{\epsilon}^{\prime}\right|^{2}+\frac{1}{r^{2}} f_{\epsilon}^{2}+\frac{1}{\epsilon^{2}}\left(1-f_{\epsilon}^{2}\right)^{2}\right\} r d r .
$$

Proposition 3.1. Let $R_{1}$ be as in (3.2) and let $\rho$ satisfy

$$
\epsilon R_{1}<\rho<1 \text {. }
$$

Then $I_{\epsilon}(\rho)$ of (3.5) admits

$$
I_{\epsilon}(\rho)=\pi \log (1 / \epsilon)+\pi \log \rho+C_{0}+O\left(\epsilon^{2} / \rho^{2}\right),
$$

where $C_{0}=C_{0}\left(R_{1}\right)$ is a constant independent of $\epsilon$ and $\rho$.

Proof. Since $f_{\epsilon}$ satisfies

$$
f_{\epsilon}^{\prime \prime}+\frac{1}{r} f_{\epsilon}^{\prime}-\frac{1}{r^{2}} f_{\epsilon}+\frac{1}{\epsilon^{2}}\left(1-f_{\epsilon}^{2}\right) f_{\epsilon}=0, \quad f_{\epsilon}(0)=0,
$$

we obtain

$$
\int_{0}^{\rho}\left(\left|f_{\epsilon}^{\prime}\right|^{2}+\frac{1}{r^{2}} f_{\epsilon}^{2}\right) r d r=\frac{1}{\epsilon^{2}} \int_{0}^{\rho}\left(1-f_{\epsilon}^{2}\right) f_{\epsilon}^{2} r d r+O\left(\epsilon^{2} / \rho^{2}\right)
$$

where we used

$$
\left[r f_{\epsilon}^{\prime} f_{\epsilon}\right]_{r=0}^{\rho}=\rho f_{\epsilon}^{\prime}(\rho) f_{\epsilon}(\rho)=O\left(\epsilon^{2} / \rho^{2}\right)
$$

(recall (3.4)). By virtue of (3.8)

$$
\begin{aligned}
I_{\epsilon}(\rho) & =\pi \int_{0}^{\rho}\left\{\frac{1}{\epsilon^{2}}\left(1-f_{\epsilon}^{2}\right) f_{\epsilon}^{2}+\frac{1}{2 \epsilon^{2}}\left(1-f_{\epsilon}^{2}\right)^{2}\right\} r d r+O\left(\epsilon^{2} / \rho^{2}\right) \\
& =\frac{\pi}{2 \epsilon^{2}} \int_{0}^{\rho}\left(1-f_{\epsilon}^{2}\right)\left(1+f_{\epsilon}^{2}\right) r d r+O\left(\epsilon^{2} / \rho^{2}\right) .
\end{aligned}
$$

Put

$$
\left\{\begin{array}{l}
I_{\epsilon}(\rho)=I_{\epsilon}^{(1)}+I_{\epsilon}^{(2)}+O\left(\epsilon^{2} / \rho^{2}\right) \\
I^{(1)}:=\frac{\pi}{2 \epsilon^{2}} \int_{0}^{\epsilon R_{1}}\left(1-f_{\epsilon}^{2}\right)\left(1+f_{\epsilon}^{2}\right) r d r \\
I^{(2)}:=\frac{\pi}{2 \epsilon^{2}} \int_{\epsilon R_{1}}^{\rho}\left(1-f_{\epsilon}^{2}\right)\left(1+f_{\epsilon}^{2}\right) r d r
\end{array}\right.
$$

We compute $I^{(1)}$ and $I^{(2)}$. By (3.3)

$$
I^{(1)}=\frac{\pi}{2} \int_{0}^{R_{1}}\left(1-\left\{f_{\infty}(s)\right\}^{2}\right)\left(1+\left\{f_{\infty}(s)\right\}^{2}\right) s d s
$$


which is independent of $\epsilon$ and $\rho$. On the other hand we can compute

$$
\begin{aligned}
I^{(2)} & =\pi \int_{\epsilon R_{1}}^{\rho}\left\{\frac{1}{\epsilon^{2}}\left(1-f_{\epsilon}^{2}\right)\left(1+f_{\epsilon}^{2}\right) / 2\right\} r d r \\
& =\pi \int_{\epsilon R_{1}}^{\rho} \frac{1}{\epsilon^{2}}\left(1-f_{\epsilon}\right)\left(1+f_{\epsilon}\right)\left(1-\epsilon^{2} / 2 r^{2}+O\left(\epsilon^{4} / r^{4}\right)\right) r d r \\
& =\pi \int_{\epsilon R_{1}}^{\rho} \frac{1}{r} d r+\pi \int_{\epsilon R_{1}}^{\rho} O\left(\epsilon^{2} / r^{3}\right) d r
\end{aligned}
$$

Thus

$$
I^{(2)}=\pi \log \rho-\pi \log \epsilon-\pi \log R_{1}+O\left(1 / R_{1}\right)+O\left(\epsilon^{2} / \rho^{2}\right) .
$$

Using (3.10) and (3.11) in (3.9), we obtain the desired (3.6).

4. An approximate solution. Given $\rho_{0}$, set

$$
Y:=\left\{\boldsymbol{y} \in \hat{\Omega}^{m}:\left|y^{(j)}-y^{(k)}\right| \geq 2 \rho_{0}, j \neq k, \quad \operatorname{dist}\left(y^{(j)}, \partial \Omega\right) \geq \frac{3}{2} \rho_{0}\right\}
$$

We take $\rho_{0}$ small so that the set $Y$ is nonempty. Throughout this section, we assume that $\rho(\epsilon)$ is a positive function satisfying

$$
\lim _{\epsilon \rightarrow 0} \rho(\epsilon)=0, \quad \lim _{\epsilon \rightarrow 0} \frac{\epsilon}{\rho(\epsilon)}=0 .
$$

We note that Propositions 2.2, 3.1 in the previous two sections still hold for putting $\rho=2 \rho(\epsilon)$.

Let $\chi$ be a function in $C^{\infty}(\mathbb{R})$ satisfying

$$
\chi(s)= \begin{cases}1, & s \leq 0 \\ 0, & s \geq 1\end{cases}
$$

and define

$$
\chi_{\epsilon}(r):=\chi(r / \rho(\epsilon)-1)
$$

Then

$$
\chi_{\epsilon}(r)= \begin{cases}1, & r \leq \rho(\epsilon) \\ 0, & 2 \rho(\epsilon) \leq r\end{cases}
$$

Set

$$
\Omega_{\rho(\epsilon)}=\Omega \backslash \cup_{j=1}^{m} \overline{B_{\rho(\epsilon)}\left(y^{(j)}\right)}, \quad \Omega_{2 \rho(\epsilon)}=\Omega \backslash \cup_{j=1}^{m} \overline{B_{2 \rho(\epsilon)}\left(y^{(j)}\right)}
$$

and for $\boldsymbol{y} \in Y$ define a positive function with zeros at $y=y^{(j)}, 1 \leq j \leq m$, as follows:

$$
w_{\epsilon}(x ; \boldsymbol{y}):=\left\{\begin{array}{cl}
g_{\epsilon}:=1-\frac{\epsilon^{2}}{2}|\nabla \Theta|^{2}, & x \in \Omega_{2 \rho(\epsilon)}, \\
g_{\epsilon}+\chi_{\epsilon}^{(j)}\left(f_{\epsilon}^{(j)}-g_{\epsilon}\right), & \rho(\epsilon) \leq\left|x-y^{(j)}\right| \leq 2 \rho(\epsilon), \quad 1 \leq j \leq m, \\
f_{\epsilon}^{(j)}, & x \in B_{\rho(\epsilon)}\left(y^{(j)}\right), \quad 1 \leq j \leq m,
\end{array}\right.
$$


where $\Theta=\Theta(x ; \boldsymbol{y})$ is defined by $(2.5)$ and

$$
f_{\epsilon}^{(j)}(x):=f_{\epsilon}\left(\left|x-y^{(j)}\right|\right), \quad \chi_{\epsilon}^{(j)}:=\chi_{\epsilon}\left(\left|x-y^{(j)}\right|\right) .
$$

We write

$$
\mathcal{F}(u):=\Delta u+\frac{1}{\epsilon^{2}}\left(1-|u|^{2}\right) u .
$$

With $u_{h}=u_{h}(x ; \boldsymbol{y})$ in $(2.6)$, define

$$
u_{\epsilon}(x ; \boldsymbol{y}):=w_{\epsilon}(x ; \boldsymbol{y}) u_{h}(x ; \boldsymbol{y}) .
$$

Then

$$
\mathcal{F}\left(u_{\epsilon}\right)=\left[\Delta w_{\epsilon}+\left\{-|\nabla \Theta|^{2}+\frac{1}{\epsilon^{2}}\left(1-w_{\epsilon}^{2}\right)\right\} w_{\epsilon}+2 i \nabla w_{\epsilon} \cdot \nabla \Theta\right] u_{h}
$$

Here we used (2.8) and

$$
\nabla u_{h}=i(\nabla \Theta) u_{h}, \quad\left|\nabla u_{h}\right|^{2}=|\nabla \Theta|^{2}, \quad x \neq y^{(j)} \quad(1 \leq j \leq m) .
$$

LEMMA 4.1. There exist a constant $C_{1}>0$ and a small number $\epsilon_{1}>0$ such that for $\epsilon \in\left(0, \epsilon_{1}\right)$,

$$
\left|\mathcal{F}\left(u_{\epsilon}\right)\right| \leq\left\{\begin{array}{l}
C_{1} \epsilon^{2}\left\{1+\sum_{j=1}^{m} \frac{1}{\left|x-y^{(j)}\right|^{4}}\right\}, \quad x \in \Omega_{2 \rho(\epsilon)}, \\
C_{1}\left\{\frac{1}{\left|x-y^{(j)}\right|}+\frac{\epsilon^{2}}{\left|x-y^{(j)}\right|^{4}}\right\}, \quad \rho(\epsilon) \leq\left|x-y^{(j)}\right| \leq 2 \rho(\epsilon), \quad 1 \leq j \leq m, \\
C_{1}\left\{\frac{f_{\epsilon}^{(j)}}{\left|x-y^{(j)}\right|}+\left|f_{\epsilon}^{\prime}\left(\left|x-y^{(j)}\right|\right)\right|\right\}, \quad x \in B_{\rho(\epsilon)}\left(y^{(j)}\right), \quad 1 \leq j \leq m .
\end{array}\right.
$$

Before proving this lemma, we estimate $L^{1}$-norm of $\mathcal{F}\left(u_{\epsilon}\right)$. Since

$$
\int_{0}^{\rho(\epsilon)}\left|f_{\epsilon}^{\prime}(r)\right| r d r=\epsilon \int_{0}^{\rho(\epsilon) / \epsilon} \frac{d}{d s} f_{\infty}(s) s d s=O(\rho(\epsilon))
$$

and

$$
\int_{0}^{\rho(\epsilon)} \frac{f_{\epsilon}(r)}{r} r d r \leq \rho(\epsilon)
$$

hold, by (4.9) of Lemma 4.1 we easily check that there is a constant $C_{1}>0$ such that

$$
\int_{\Omega_{\rho(\epsilon)}}\left|\mathcal{F}\left(u_{\epsilon}\right)\right| d x \leq C_{1} \epsilon^{2} /(\rho(\epsilon))^{2},
$$

and

$$
\int_{B_{\rho(\epsilon)}\left(y^{(j)}\right)}\left|\mathcal{F}\left(u_{\epsilon}\right)\right| d x \leq C_{1} \rho(\epsilon)
$$


for each $j$. Moreover we can similarly estimate the derivative of $\mathcal{F}\left(u_{\epsilon}\right)$ with respect to $y_{p}^{(k)}$ to obtain

$$
\int_{\Omega}\left|\frac{\partial}{\partial y_{p}^{(k)}} \mathcal{F}\left(u_{\epsilon}\right)\right| d x \leq C_{1} \max \left\{\log (1 / \epsilon), \epsilon^{2} / \rho(\epsilon)^{3}\right\}
$$

Hence we have

LEMMA 4.2. Let $\epsilon_{1}$ be a number as in Lemma 4.1. Then there is a constant $C_{1}$ such that for $\epsilon \in\left(0, \epsilon_{1}\right)$,

$$
\begin{aligned}
& \int_{\Omega}\left|\mathcal{F}\left(u_{\epsilon}\right)\right| d x \leq C_{1} \max \left\{\rho(\epsilon), \epsilon^{2} / \rho(\epsilon)^{2}\right\} \\
& \int_{\Omega}\left|\frac{\partial}{\partial y_{p}^{(k)}} \mathcal{F}\left(u_{\epsilon}\right)\right| d x \leq C_{1} \max \left\{\log (1 / \epsilon), \epsilon^{2} / \rho(\epsilon)^{3}\right\}
\end{aligned}
$$

Proof of Lemma 4.1 : Henceforth we often simply write $\rho$ for $\rho(\epsilon)$ if there is no confusion.

First we check

$$
\begin{aligned}
|\nabla \Theta|^{2} & \leq\left|\sum_{j=1}^{m} d_{j} \nabla_{x}\left(\operatorname{Arg}\left(x-y^{(j)}\right)+\varphi\left(x ; y^{(j)}\right)\right)\right|^{2} \\
& \leq C_{1}\left\{1+\sum_{j=1}^{m} \frac{1}{\left|x-y^{(j)}\right|^{2}}\right\}, \\
\left.|\Delta| \nabla \Theta\right|^{2} \mid & \leq C_{1}\left\{1+\sum_{j=1}^{m} \frac{1}{\left|x-y^{(j)}\right|^{4}}\right\}, \\
\left.|| \nabla \Theta\right|^{2}-\frac{1}{\left|x-y^{(j)}\right|^{2}} \mid & \leq C_{1}\left\{1+\sum_{j=1}^{m} \frac{1}{\left|x-y^{(j)}\right|}\right\}, \quad x \in B_{\rho}\left(y^{(j)}\right) .
\end{aligned}
$$

For $\rho \leq\left|x-y^{(j)}\right| \leq 2 \rho$, by (3.4)

$$
f_{\epsilon}^{(j)}-g_{\epsilon}=\frac{\epsilon^{2}}{2}\left(|\nabla \Theta|^{2}-1 /\left|x-y^{(j)}\right|^{2}\right)+O\left(\epsilon^{4} /\left|x-y^{(j)}\right|^{4}\right),
$$

thus

$$
\left|f_{\epsilon}^{(j)}-g_{\epsilon}\right| \leq \frac{C_{1} \epsilon^{2}}{\left|x-y^{(j)}\right|}, \quad \rho \leq\left|x-y^{(j)}\right| \leq 2 \rho .
$$

We also see that

$$
-|\nabla \Theta|^{2}+\frac{1}{\epsilon^{2}}\left(1-g_{\epsilon}^{2}\right)=-\frac{\epsilon^{2}}{4}|\nabla \Theta|^{2}, \quad x \in \Omega_{2 \rho}
$$


and

$$
\begin{aligned}
& -|\nabla \Theta|^{2}+\frac{1}{\epsilon^{2}}\left(1-w_{\epsilon}^{2}\right) \\
= & -|\nabla \Theta|^{2}+\frac{1}{\epsilon^{2}}\left(1-g_{\epsilon}^{2}\right)-\chi_{\epsilon}^{(j)}\left(1+g_{\epsilon}\right)\left(f_{\epsilon}^{(j)}-g_{\epsilon}\right) / \epsilon^{2} \\
& +\chi_{\epsilon}^{(j)}\left(1-g_{\epsilon}\right)\left(f_{\epsilon}^{(j)}-g_{\epsilon}\right) / \epsilon^{2}-\left(\chi_{\epsilon}^{(j)}\right)^{2}\left(f_{\epsilon}^{(j)}-g_{\epsilon}\right)^{2} / \epsilon^{2}, \quad \rho \leq\left|x-y^{(j)}\right| \leq 2 \rho,
\end{aligned}
$$

from which

$$
\left.|-| \nabla \Theta\right|^{2}+\frac{1}{\epsilon^{2}}\left(1-w_{\epsilon}^{2}\right) \mid \leq\left\{\begin{array}{l}
C_{1} \epsilon^{2}\left\{1+\sum_{j=1}^{m} \frac{1}{\left|x-y^{(j)}\right|^{4}}\right\}, x \in \Omega_{2 \rho}, \\
C_{1}\left\{\frac{1}{\left|x-y^{(j)}\right|}+\frac{\epsilon^{2}}{\left|x-y^{(j)}\right|^{4}}\right\}, \\
\rho \leq\left|x-y^{(j)}\right| \leq 2 \rho
\end{array}\right.
$$

follows. By (4.13) and (4.16), we obtain

$$
\left|\Delta w_{\epsilon}+\left\{-|\nabla \Theta|^{2}+\frac{1}{\epsilon^{2}}\left(1-w_{\epsilon}^{2}\right)\right\} w_{\epsilon}\right| \leq C_{1} \epsilon^{2}\left\{1+\sum_{j=1}^{m} \frac{1}{\left|x-y^{(j)}\right|^{4}}\right\}, \quad x \in \Omega_{2 \rho} .
$$

Moreover combining (4.16) with

$$
\begin{aligned}
\left|\Delta w_{\epsilon}\right| & \leq\left|\Delta g_{\epsilon}\right|+\left|\Delta \chi_{\epsilon}^{(j)}\right|\left|f_{\epsilon}^{(j)}-g_{\epsilon}\right|+2\left|\nabla \chi_{\epsilon}^{(j)}\right|\left|\nabla\left(f_{\epsilon}^{(j)}-g_{\epsilon}\right)\right|+\left|\Delta\left(f_{\epsilon}^{(j)}-g_{\epsilon}\right)\right| \\
& \leq \frac{C_{1} \epsilon^{2}}{\left|x-y^{(j)}\right|^{4}}+\frac{C_{1} \epsilon^{2}}{\rho^{2}\left|x-y^{(j)}\right|}+\frac{C_{1} \epsilon^{2}}{\rho\left|x-y^{(j)}\right|^{2}}+\frac{C_{1} \epsilon^{2}}{\left|x-y^{(j)}\right|^{3}} \\
& \leq \frac{C_{1} \epsilon^{2}}{\left|x-y^{(j)}\right|^{4}}, \quad \rho \leq\left|x-y^{(j)}\right| \leq 2 \rho,
\end{aligned}
$$

we get

$$
\left|\Delta w_{\epsilon}+\left\{-|\nabla \Theta|^{2}+\frac{1}{\epsilon^{2}}\left(1-w_{\epsilon}^{2}\right)\right\} w_{\epsilon}\right| \leq C_{1}\left\{\frac{1}{\left|x-y^{(j)}\right|}+\frac{\epsilon^{2}}{\left|x-y^{(j)}\right|^{4}}\right\}
$$

for $\rho \leq\left|x-y^{(j)}\right| \leq 2 \rho$.

On the other hand we can compute

$$
\nabla w_{\epsilon} \cdot \nabla \Theta=\left\{\begin{array}{l}
\nabla g_{\epsilon} \cdot \nabla \Theta=-\frac{\epsilon^{2}}{2} \nabla\left(|\nabla \Theta|^{2}\right) \cdot \nabla \Theta, \quad x \in \Omega_{2 \rho}, \\
\left\{\nabla g_{\epsilon}+\left(f_{\epsilon}^{(j)}-g_{\epsilon}\right) \nabla \chi_{\epsilon}^{(j)}+\chi_{\epsilon}^{(j)} \nabla\left(f_{\epsilon}^{(j)}-g_{\epsilon}\right)\right\} \cdot \nabla \Theta, \\
\rho \leq\left|x-y^{(j)}\right| \leq 2 \rho .
\end{array}\right.
$$

Thus we obtain

$$
\left|\nabla w_{\epsilon} \cdot \nabla \Theta\right| \leq \epsilon^{2} C_{1}\left\{1+\sum_{j=1}^{m} \frac{1}{\left|x-y^{(j)}\right|^{4}}\right\}, \quad x \in \Omega_{2 \rho} .
$$


Furthermore, with the aid of

$$
\nabla_{x}\left|x-y^{(j)}\right| \cdot \operatorname{Arg}_{x}\left(x-y^{(j)}\right)=0
$$

we can show

$$
\left|\nabla \chi_{\epsilon}^{(j)} \cdot \nabla \Theta\right| \leq C_{1} / \rho
$$

Hence we have

$$
\begin{aligned}
\left|\nabla w_{\epsilon} \cdot \nabla \Theta\right| & \leq C_{1}\left\{\frac{\epsilon^{2}}{\left|x-y^{(j)}\right|^{4}}+\frac{\epsilon^{2}}{\rho\left|x-y^{(j)}\right|}+\frac{\epsilon^{2}}{\left|x-y^{(j)}\right|^{3}}\right\} \\
& \leq \frac{C_{1} \epsilon^{2}}{\left|x-y^{(j)}\right|^{4}}, \quad \rho \leq\left|x-y^{(j)}\right| \leq 2 \rho .
\end{aligned}
$$

Finally we compute

$$
\begin{aligned}
\mathcal{F}\left(u_{\epsilon}\right) & =\left\{\Delta f_{\epsilon}^{(j)}-|\nabla \Theta|^{2} f_{\epsilon}^{(j)}+\frac{1}{\epsilon^{2}}\left(1-\left(f_{\epsilon}^{(j)}\right)^{2}\right) f_{\epsilon}^{(j)}+2 i \nabla f_{\epsilon}^{(j)} \cdot \nabla \Theta\right\} e^{i \Theta} \\
& =-\left\{\left(|\nabla \Theta|^{2}-\frac{1}{\left|x-y^{(j)}\right|^{2}}\right) f_{\epsilon}^{(j)}+2 i \nabla f_{\epsilon}^{(j)} \cdot \nabla \Theta\right\} e^{i \Theta}, \quad x \in B_{\rho}\left(y^{(j)}\right) .
\end{aligned}
$$

Since

$$
\nabla f_{\epsilon}^{(j)} \cdot \nabla \Theta=f_{\epsilon}^{\prime}\left(\left|x-y^{(j)}\right|\right) \frac{x-y^{(j)}}{\left|x-y^{(j)}\right|} \cdot\left\{\nabla \Theta-\frac{\left(x-y^{(j)}\right)^{\perp}}{\left|x-y^{(j)}\right|^{2}}\right\}
$$

we obtain

$$
\left|\mathcal{F}\left(u_{\epsilon}\right)\right| \leq C_{1}\left\{f_{\epsilon}^{(j)} /\left|x-y^{(j)}\right|+\left|f_{\epsilon}^{\prime}\left(\left|x-y^{(j)}\right|\right)\right|\right\}, \quad x \in B_{\rho}\left(y^{(j)}\right) .
$$

In terms of (4.17) (4.18), (4.19), (4.20) and (4.21) we get to the desired estimate (4.9). ㅁ

Next we show some estimates for derivatives of the approximate solution for later arguments. Let $\langle u, v\rangle$ denotes

$$
\langle u, v\rangle_{L^{2}}=\operatorname{Re} \int_{\Omega} u(x) \overline{v(x)} d x
$$

for $u, v \in L^{2}(\Omega ; \mathbb{C})$. Recall that $\mathbb{C}$ is identified with $\mathbb{R}^{2}$, therefore this is the inner product in $L^{2}\left(\Omega ; \mathbb{R}^{2}\right)$.

LEMMA 4.3.

$$
\begin{gathered}
\int_{\Omega}\left|\frac{\partial u_{\epsilon}}{\partial y_{p}^{(k)}}\right| d x=O(1), \\
\left\langle\frac{\partial u_{\epsilon}}{\partial y_{p}^{(k)}}, \frac{\partial u_{\epsilon}}{\partial y_{q}^{(j)}}\right\rangle_{L^{2}}= \begin{cases}\pi \log (1 / \epsilon)+O(1), & k=j \text { and } p=q, \\
O(1), & k \neq j \text { or } p \neq q,\end{cases}
\end{gathered}
$$


and

$$
\int_{\Omega}\left|\frac{\partial^{2} u_{\epsilon}}{\partial y_{p}^{(k)} y_{q}^{(j)}}\right| d x=O(\log (1 / \epsilon)), \quad 1 \leq k, j \leq m, \quad 1 \leq p, q \leq 2
$$

Proof. The estimate (4.23) is easily verified, therefore we prove (4.24). First we write

$$
\left\langle\frac{\partial u_{\epsilon}}{\partial y_{p}^{(k)}}, \frac{\partial u_{\epsilon}}{\partial y_{q}^{(j)}}\right\rangle_{L^{2}}=\int_{\Omega}\left(\frac{\partial w_{\epsilon}}{\partial y_{p}^{(k)}} \frac{\partial w_{\epsilon}}{\partial y_{q}^{(j)}}+w_{\epsilon}^{2} \frac{\partial \Theta}{\partial y_{p}^{(k)}} \frac{\partial \Theta}{\partial y_{q}^{(j)}}\right) d x
$$

Since

$$
\frac{\partial w_{\epsilon}}{\partial y_{p}^{(k)}}=\left\{\begin{array}{l}
-\frac{\epsilon^{2}}{2} \frac{\partial}{\partial y_{p}^{(k)}}|\nabla \Theta|^{2}, \quad x \in \Omega_{2 \rho}, \\
-\frac{\epsilon^{2}}{2} \frac{\partial}{\partial y_{p}^{(k)}}|\nabla \Theta|^{2}+\frac{\partial}{\partial y_{p}^{(k)}} \chi_{\epsilon}^{(j)}\left(f_{\epsilon}^{(j)}-g_{\epsilon}\right) \\
+\chi_{\epsilon}^{(j)} \frac{\partial}{\partial y_{p}^{(k)}}\left(f_{\epsilon}^{(j)}-g_{\epsilon}\right), \quad \rho \leq\left|x-y^{(j)}\right| \leq 2 \rho, \\
\frac{\partial}{\partial y_{p}^{(k)}} f_{\epsilon}^{(j)}, \quad x \in B_{\rho}\left(y^{(j)}\right),
\end{array}\right.
$$

we obtain

$$
\int_{\Omega}\left|\frac{\partial w_{\epsilon}}{\partial y_{p}^{(k)}}\right|^{2} d x \leq C_{1} \int_{\Omega_{\rho}} \frac{\epsilon^{4}}{\left|x-y^{(j)}\right|^{6}}+\int_{B_{\rho}\left(y^{(k)}\right)}\left|f_{\epsilon}^{\prime}\left(\left|x-y^{(j)}\right|\right)\right|^{2} d x+O(1) .
$$

Therefore from a simple computation we can see

$$
\int_{\Omega}\left|\frac{\partial w_{\epsilon}}{\partial y_{p}^{(k)}}\right|^{2} d x=O(1)
$$

We also easily check

$$
\int_{\Omega} \frac{\partial w_{\epsilon}}{\partial y_{p}^{(k)}} \frac{\partial w_{\epsilon}}{\partial y_{q}^{(j)}} d x=O(1)
$$

Next we compute

$$
\int_{\Omega} w_{\epsilon}^{2} \frac{\partial \Theta}{\partial y_{p}^{(k)}} \frac{\partial \Theta}{\partial y_{q}^{(j)}} d x
$$

First consider the case for $k=j$ and $p=q=1$. Then

$$
\frac{\partial \Theta}{\partial y_{1}^{(k)}}=d_{k}\left\{-\frac{\left(x_{2}-y_{2}^{(k)}\right)}{\left|x-y^{(k)}\right|^{2}}+\frac{\partial}{\partial y_{1}^{(k)}} \varphi\left(x ; y^{(k)}\right)\right\}
$$


by which

$$
\begin{aligned}
\int_{\Omega_{\rho}} w_{\epsilon}^{2}\left|\frac{\partial \Theta}{\partial y_{1}^{(k)}}\right|^{2} d x & =\int_{0}^{2 \pi} \int_{\rho}^{\rho_{0}} \frac{r^{2} \sin ^{2} \theta}{r^{4}} r d r d \theta+O(1) \\
& =\pi\left(\log \rho_{0}-\log \rho\right)+O(1)
\end{aligned}
$$

and

$$
\begin{aligned}
\int_{B_{\rho}\left(y^{(k)}\right)}\left(f_{\epsilon}^{(k)}\right)^{2}\left|\frac{\partial \Theta}{\partial y_{1}^{(k)}}\right|^{2} d x & =\int_{0}^{2 \pi} \int_{0}^{\rho}\left(f_{\epsilon}(r)\right)^{2} \frac{\sin ^{2} \theta}{r} d r d \theta+O(1) \\
& =\pi \int_{0}^{\rho / \epsilon} \frac{\left(f_{\infty}(s)\right)^{2}}{s} d s+O(1) \\
& =\pi(\log \rho-\log \epsilon)+O(1) .
\end{aligned}
$$

Thus recalling that $\rho=\rho(\epsilon)$ satisfying (4.2) and $\rho_{0}$ is a fixed number, we obtain

$$
\int_{\Omega} w_{\epsilon}^{2}\left|\frac{\partial \Theta}{\partial y_{1}^{(k)}}\right|^{2} d x=\pi \log (1 / \epsilon)+O(1)
$$

It follows from (4.26) and (4.28) that

$$
\int_{\Omega}\left|\frac{\partial u_{\epsilon}}{\partial y_{1}^{(k)}}\right|^{2} d x=\pi \log (1 / \epsilon)+O(1)
$$

Similarly we obtain the same estimate for $k=j$ and $p=q=2$.

Next we consider the case for $k=j$ and $p \neq q$. For simplicity, we set $p=1, q=2$. Then

$$
\frac{\partial \Theta}{\partial y_{1}^{(k)}} \frac{\partial \Theta}{\partial y_{2}^{(k)}}=\frac{-\left(x_{2}-y_{2}^{(k)}\right)}{\left|x-y^{(k)}\right|^{2}} \frac{\left(x_{1}-y_{1}^{(k)}\right)}{\left|x-y^{(k)}\right|^{2}}+O\left(1 /\left|x-y^{(k)}\right|\right), \quad x \approx y^{(k)} .
$$

Because of

$$
\int_{B_{\rho}}\left(f_{\epsilon}^{(k)}\right)^{2} \frac{-\left(x_{2}-y_{2}^{(k)}\right)}{\left|x-y^{(k)}\right|^{2}} \frac{\left(x_{1}-y_{1}^{(k)}\right)}{\left|x-y^{(k)}\right|^{2}} d x=\int_{0}^{2 \pi} \int_{0}^{\rho} \frac{-\sin \theta \cos \theta}{r} f_{\epsilon}^{2} d r d \theta=0
$$

one can easily check

$$
\int_{\Omega} w_{\epsilon}^{2} \frac{\partial \Theta}{\partial y_{1}^{(k)}} \frac{\partial \Theta}{\partial y_{2}^{(k)}}=O(1)
$$

As for the remaining cases, $k \neq j$, we easily verify

$$
\frac{\partial \Theta}{\partial y_{p}^{(k)}} \frac{\partial \Theta}{\partial y_{q}^{(j)}}=O\left(1 /\left|x-y^{(j)}\right|\right), \quad x \approx y^{(j)}
$$

from which

$$
\int_{\Omega} w_{\epsilon}^{2} \frac{\partial \Theta}{\partial y_{p}^{(k)}} \frac{\partial \Theta}{\partial y_{q}^{(j)}}=O(1)
$$

follows. This concludes the proof of (4.24).

To prove (4.25), we repeat the similar computations done for (4.24). We leave it to the readers. 
5. Energy for the approximate solution. The following lemma involves the closeness between the energy of $u_{\epsilon}$ and the renormalized energy $V(\boldsymbol{y})$ :

Lemma 5.1. Let $E_{\epsilon}(u)$ be the energy defined by (1.3). Assume (4.2). Then for small $\epsilon>0, E_{\epsilon}\left(u_{\epsilon}(\cdot ; \boldsymbol{y})\right)$ is $C^{1}$ in $\boldsymbol{y}$ and

$$
E_{\epsilon}\left(u_{\epsilon}\right)=m \pi \log (1 / \epsilon)+C_{0}+V(\boldsymbol{y})+O(\rho(\epsilon))+O\left(\epsilon^{2} / \rho(\epsilon)^{2}\right) .
$$

Moreovery if $\epsilon^{2} / \rho(\epsilon)^{3} \rightarrow 0$ as $\epsilon \rightarrow 0$, then

$$
\frac{\partial}{\partial y_{p}^{(j)}} E_{\epsilon}\left(u_{\epsilon}\right)=\frac{\partial}{\partial y_{p}^{(j)}} V(\boldsymbol{y})+O(\rho(\epsilon))+O\left(\epsilon^{2} / \rho(\epsilon)^{3}\right) .
$$

holds.

Proof. Since the proof of the former part is simple, we focus on (5.2). For simplicity of notation we simply write $\rho$ for $\rho(\epsilon)$ again. Set

$$
e_{\epsilon}\left(u_{\epsilon}\right):=\left|\nabla w_{\epsilon}\right|^{2}+w_{\epsilon}^{2}\left|\nabla u_{h}\right|^{2}+\frac{1}{2 \epsilon^{2}}\left(1-w_{\epsilon}^{2}\right)^{2} .
$$

Then

$$
\begin{aligned}
\frac{\partial}{\partial y_{p}^{(j)}} E_{\epsilon}\left(u_{\epsilon}\right) & =\frac{1}{2} \int_{\Omega} \frac{\partial}{\partial y_{p}^{(j)}} e_{\epsilon}\left(u_{\epsilon}\right) d x \\
& =\frac{1}{2} \int_{\Omega_{\rho}} \frac{\partial}{\partial y_{p}^{(j)}} e_{\epsilon}\left(u_{\epsilon}\right) d x+\frac{1}{2} \sum_{k=1}^{m} \int_{B_{\rho}\left(y^{(k)}\right)} \frac{\partial}{\partial y_{p}^{(j)}} e_{\epsilon}\left(u_{\epsilon}\right) d x
\end{aligned}
$$

We write

$$
\begin{aligned}
& e_{\epsilon}\left(u_{\epsilon}\right)=\left|\nabla u_{h}\right|^{2}+W_{\epsilon}, \quad x \in \Omega_{\rho} \\
& W_{\epsilon}:=\left|\nabla w_{\epsilon}\right|^{2}+\left(1-w_{\epsilon}^{2}\right)\left\{-\left|\nabla u_{h}\right|^{2}+\frac{1}{2 \epsilon^{2}}\left(1-w_{\epsilon}^{2}\right)\right\}, \quad x \in \Omega_{\rho},
\end{aligned}
$$

and

$$
\left.e_{\epsilon}\left(u_{\epsilon}\right)=\left|\nabla f_{\epsilon}^{(k)}\right|^{2}+\left(f_{\epsilon}^{(k)}\right)^{2}\left|\nabla u_{h}\right|^{2}+\frac{1}{2 \epsilon^{2}}\left(1-\left(f_{\epsilon}^{(k)}\right)^{2}\right)\right)^{2}
$$

$$
=e_{\epsilon}\left(f_{\epsilon}^{(k)} e^{i \operatorname{Arg}\left(x-y^{(k)}\right)}\right)+\left|\nabla u_{h}\right|^{2}-\frac{1}{\left|x-y^{(k)}\right|^{2}}+R_{\epsilon}^{(k)}, \quad x \in B_{\rho}\left(y^{(k)}\right),
$$

where we put

$$
R_{\epsilon}^{(k)}:=\left(\left(f_{\epsilon}^{(k)}\right)^{2}-1\right)\left\{\left|\nabla u_{h}\right|^{2}-\frac{1}{\left|x-y^{(k)}\right|^{2}}\right\} .
$$

Note that

$$
e_{\epsilon}\left(f_{\epsilon}^{(k)} e^{i \operatorname{Arg}\left(x-y^{(k)}\right)}\right)=\left|\nabla f_{\epsilon}^{(k)}\right|^{2}+\left(f_{\epsilon}^{(k)}\right)^{2} /\left|x-y^{(k)}\right|^{2}+\frac{1}{2 \epsilon^{2}}\left(1-\left(f_{\epsilon}^{(k)}\right)^{2}\right)^{2} .
$$


If we prove

$$
\begin{aligned}
& \int_{B_{\rho}\left(y^{(k)}\right)} \frac{\partial}{\partial y_{p}^{(j)}} e_{\epsilon}\left(f_{\epsilon}^{(k)} e^{i \operatorname{Arg}\left(x-y^{(k)}\right)}\right) d x=0 \\
& \left|\sum_{k=1}^{m} \int_{B_{\rho}\left(y^{(k)}\right)} \frac{\partial}{\partial y_{p}^{(j)}} R_{\epsilon}^{(k)} d x\right|=O\left(\epsilon^{2} / \rho^{2}\right)+O(\rho) \\
& \left|\int_{\Omega_{\rho}} \frac{\partial}{\partial y_{p}^{(j)}} W_{\epsilon} d x\right|=O\left(\epsilon^{2} / \rho^{3}\right)
\end{aligned}
$$

and

$$
\begin{aligned}
& \frac{1}{2} \int_{\Omega_{\rho}} \frac{\partial}{\partial y_{p}^{(j)}}\left|\nabla u_{h}\right|^{2} d x+\frac{1}{2} \sum_{k=1}^{m} \int_{B_{\rho}\left(y^{(k)}\right)} \frac{\partial}{\partial y_{p}^{(j)}}\left\{\left|\nabla u_{h}\right|^{2}-\frac{1}{\left|x-y^{(k)}\right|^{2}}\right\} d x \\
= & \frac{\partial}{\partial y_{p}^{(j)}} V(\boldsymbol{y})+O(\rho),
\end{aligned}
$$

then it is clear that the desired (5.2) follows. Henceforth we fix $p=1$. For $p=2$ the same computation works, so omit it.

First consider (5.6). It is trivial that it holds for $k \neq j$. For $k=j$ it follows from

$$
\begin{aligned}
& \int_{B_{\rho}\left(y^{(j)}\right)} \frac{\partial}{\partial y_{1}^{(j)}} e_{\epsilon}\left(f_{\epsilon}^{(j)} e^{i \operatorname{Arg}\left(x-y^{(j)}\right)}\right) d x \\
= & \int_{B_{\rho}\left(y^{(j)}\right)}-\frac{\partial}{\partial x_{1}^{(j)}} e_{\epsilon}\left(f_{\epsilon}^{(j)} e^{i \operatorname{Arg}\left(x-y^{(j)}\right)}\right) d x \\
= & \int_{0}^{2 \pi}-\left\{\left|f_{\epsilon}^{\prime}(\rho)\right|^{2}+f_{\epsilon}(\rho)^{2} / \rho^{2}+\frac{1}{2 \epsilon^{2}}\left(1-f_{\epsilon}(\rho)^{2}\right)^{2}\right\} \rho \cos \theta d \theta=0 .
\end{aligned}
$$

Second we prove (5.7). For $k \neq j$,

$$
\int_{B_{\rho}\left(y^{(k)}\right)}\left|\frac{\partial R_{\epsilon}^{(k)}}{\partial y_{1}^{(j)}}\right| d x \leq \int_{B_{\rho}\left(y^{(k)}\right)} \frac{C_{1}}{\left|x-y^{(k)}\right|} d x=O(\rho) .
$$

On the other hand for $k=j$ we need a more careful computation. Let

$$
\begin{aligned}
& \tilde{R}_{\epsilon}^{(j)}:=2\left(\left(f_{\epsilon}^{(j)}\right)^{2}-1\right) \sum_{\ell \neq j} \nabla_{x} \log \left|x-y^{(j)}\right| \cdot \nabla_{x} S\left(x, y^{(\ell)}\right), \\
& \hat{R}_{\epsilon}^{(j)}:=R_{\epsilon}^{(j)}-\tilde{R}_{\epsilon}^{(j)} .
\end{aligned}
$$

Then we easily see

$$
\frac{\partial}{\partial y_{1}^{(j)}} \tilde{R}_{\epsilon}^{(j)}=-\frac{\partial}{\partial x_{1}^{(j)}} \tilde{R}_{\epsilon}^{(j)}+2\left(\left(f_{\epsilon}^{(j)}\right)^{2}-1\right) \sum_{\ell \neq j} \nabla_{x} \log \left|x-y^{(j)}\right| \cdot \nabla_{x} \frac{\partial}{\partial x_{1}^{(j)}} S\left(x, y^{(\ell)}\right)
$$

With the aid of this identity we compute

$$
\int_{B_{\rho}\left(y^{(j)}\right)} \frac{\partial}{\partial y_{1}^{(j)}} R_{\epsilon}^{(j)} d x
$$




$$
\begin{aligned}
& =\int_{B_{\rho}\left(y^{(j)}\right)}-\frac{\partial}{\partial x_{1}^{(j)}} \tilde{R}_{\epsilon}^{(j)} d x+O(\rho) \\
& \left.=2 \int_{0}^{2 \pi}\left\{\left(f_{\epsilon}(\rho)\right)^{2}-1\right) \sum_{\ell \neq j}(\cos \theta, \sin \theta) \cdot \nabla_{x} S\left(y^{(j)}+\rho e^{i \theta}\right)\right\} d \theta+O(\rho) \\
& =O\left(\epsilon^{2} / \rho^{2}\right)+O(\rho)
\end{aligned}
$$

(we used (3.4)).

As for (5.8), we verify that leading terms of $W_{\epsilon}$ are as follows:

$$
W_{\epsilon}=C_{1} \sum_{k=1}^{m}\left\{\frac{\epsilon^{4}}{\left|x-y^{(k)}\right|^{6}}+\frac{\epsilon^{2}}{\left|x-y^{(k)}\right|^{4}}\right\}+\cdots, \quad x \in \Omega_{\rho} .
$$

Thus

$$
\left|\frac{\partial}{\partial y_{1}^{(j)}} W_{\epsilon}\right| \leq \frac{C_{1} \epsilon^{2}}{\left|x-y^{(j)}\right|^{5}}
$$

which yields (5.8).

Finally we prove (5.9). Recall

$$
\left|\nabla u_{h}\right|^{2}=\sum_{j=1}^{m} \sum_{\ell=1}^{m} \nabla_{x} G\left(x, y^{(j)}\right) \cdot \nabla_{x} G\left(x, y^{(\ell)}\right)
$$

(see (2.22)). We obtain

$$
\begin{aligned}
\frac{\partial}{\partial y_{1}^{(j)}}\left|\nabla u_{h}\right|^{2}=2 \nabla_{x} & \frac{\partial}{\partial y_{1}^{(j)}} G\left(x, y^{(j)}\right) \cdot \nabla_{x} G\left(x, y^{(j)}\right) \\
& +2 \sum_{\ell \neq j} d_{j} d_{\ell} \nabla_{x} \frac{\partial}{\partial y_{1}^{(j)}} G\left(x, y^{(j)}\right) \cdot \nabla_{x} G\left(x, y^{(\ell)}\right) .
\end{aligned}
$$

We use the similar computation done in Lemma 2.3. In fact

$$
\begin{aligned}
& \frac{1}{2} \int_{\Omega_{\rho}} \frac{\partial}{\partial y_{1}^{(j)}}\left|\nabla u_{h}\right|^{2} d x \\
= & -\sum_{k=1}^{m} \int_{\partial B_{\rho}\left(y^{(k)}\right)} \frac{\partial}{\partial y_{1}^{(j)}} G\left(x, y^{(j)}\right) \frac{\partial}{\partial \nu_{x}} G\left(x, y^{(j)}\right) d \sigma_{x} \\
& \quad-\sum_{\ell \neq j} d_{j} d_{\ell} \sum_{k=1}^{m} \int_{\partial B_{\rho}\left(y^{(k)}\right)} \frac{\partial}{\partial y_{1}^{(j)}} G\left(x, y^{(j)}\right) \frac{\partial}{\partial \nu_{x}} G\left(x, y^{(\ell)}\right) d \sigma_{x}
\end{aligned}
$$

where we noticed

$$
\frac{\partial}{\partial y_{1}^{(j)}} G\left(x, y^{(j)}\right)=0, \quad x \in \partial \Omega
$$

Moreover since

$$
\int_{\partial B_{\rho}\left(y^{(k)}\right)} \frac{\partial}{\partial y_{1}^{(j)}} \log \left|x-y^{(j)}\right| \frac{\partial}{\partial \nu_{x}} \log \left|x-y^{(j)}\right| d \sigma_{x}=-\int_{0}^{2 \pi} \frac{\cos \theta}{\rho} d \theta=0
$$


we have

$$
\begin{aligned}
& \text { (5.14) } \frac{1}{2} \int_{\Omega_{\rho}} \frac{\partial}{\partial y_{1}^{(j)}}\left|\nabla u_{h}\right|^{2} d x \\
&=-\sum_{k=1}^{m} \int_{\partial B_{\rho}\left(y^{(k)}\right)}\left\{\frac{\partial}{\partial y_{1}^{(j)}} \log \left|x-y^{(j)}\right| \frac{\partial}{\partial \nu_{x}} S\left(x, y^{(j)}\right)+\frac{\partial}{\partial y_{1}^{(j)}} S\left(x, y^{(j)}\right) \frac{\partial}{\partial \nu_{x}} G\left(x, y^{(j)}\right)\right\} d \sigma_{x} \\
& \quad-\sum_{k=1}^{m} \sum_{\ell \neq j} d_{j} d_{\ell} \int_{\partial B_{\rho}\left(y^{(k)}\right)} \frac{\partial}{\partial y_{1}^{(j)}} G\left(x, y^{(j)}\right) \frac{\partial}{\partial \nu_{x}} G\left(x, y^{(\ell)}\right) d \sigma_{x} .
\end{aligned}
$$

Similarly we can compute

$$
\frac{1}{2} \int_{B_{\rho}\left(y^{(j)}\right)} \frac{\partial}{\partial y_{1}^{(j)}}\left\{\left|\nabla u_{h}\right|^{2}-\frac{1}{\left|x-y^{(j)}\right|^{2}}\right\} d x=-2 \pi \frac{\partial}{\partial y_{1}^{(j)}} S\left(y^{(j)}, y^{(j)}\right)
$$

$$
\begin{aligned}
& +\int_{\partial B_{\rho}\left(y^{(k)}\right)}\left\{\frac{\partial}{\partial y_{1}^{(j)}} \log \left|x-y^{(j)}\right| \frac{\partial}{\partial \nu_{x}} S\left(x, y^{(j)}\right)+\frac{\partial}{\partial y_{1}^{(j)}} S\left(x, y^{(j)}\right) \frac{\partial}{\partial \nu_{x}} G\left(x, y^{(j)}\right)\right\} d \sigma_{x} \\
& +\sum_{\ell \neq j} d_{j} d_{\ell} \int_{\left|x-y^{(j)}\right|=\rho} \frac{\partial}{\partial y_{1}^{(j)}} G\left(x, y^{(j)}\right) \frac{\partial}{\partial \nu_{x}} G\left(x, y^{(\ell)}\right) d \sigma_{x} .
\end{aligned}
$$

On the other hand for $k \neq j$, because of

$$
\begin{aligned}
& \frac{\partial}{\partial y_{1}^{(j)}}\left\{\left|\nabla u_{h}\right|^{2}-\frac{1}{\left|x-y^{(j)}\right|^{2}}\right\} \\
= & \frac{\partial}{\partial y_{1}^{(j)}}\left\{\left|\nabla_{x} G\left(x, y^{(j)}\right)\right|^{2}+2 \sum_{\ell \neq j} d_{j} d_{\ell} \nabla_{x} G\left(x, y^{(j)}\right) \cdot \nabla_{x} G\left(x, y^{(\ell)}\right)\right\}
\end{aligned}
$$

and considering $\left|\nabla_{x} G\left(x, y^{(j)}\right)\right|^{2}$ is smooth in $B_{\rho}\left(y^{(k)}\right)$, one can check

$$
\begin{aligned}
& \frac{1}{2} \int_{B_{\rho}\left(y^{(k)}\right)} \frac{\partial}{\partial y_{1}^{(k)}}\left\{\left|\nabla u_{h}\right|^{2}-\frac{1}{\left|x-y^{(j)}\right|^{2}}\right\} d x \\
= & \sum_{\ell \neq j} \int_{B_{\rho}\left(y^{(k)}\right)} d_{j} d_{\ell} \nabla_{x} \frac{\partial}{\partial y_{1}^{(j)}} G\left(x, y^{(j)}\right) \cdot \nabla_{x} G\left(x, y^{(\ell)}\right) d x+O(\rho) \\
= & \sum_{\ell \neq j} \int_{\partial B_{\rho}\left(y^{(k)}\right)} d_{j} d_{\ell} \frac{\partial}{\partial y_{1}^{(j)}} G\left(x, y^{(j)}\right) \frac{\partial}{\partial \nu_{x}} G\left(x, y^{(\ell)}\right) d \sigma_{x} \\
& -2 \pi d_{j} d_{k} \frac{\partial}{\partial y_{1}^{(j)}} G\left(y^{(k)}, y^{(j)}\right)+O(\rho) .
\end{aligned}
$$

Combining (5.15) with (5.16) yields

$$
\begin{aligned}
& \text { (5.17) } \frac{1}{2} \sum_{k=1}^{m} \int_{B_{\rho}\left(y^{(k)}\right)} \frac{\partial}{\partial y_{1}^{(k)}}\left\{\left|\nabla u_{h}\right|^{2}-\frac{1}{\left|x-y^{(j)}\right|^{2}}\right\} d x \\
& =\int_{\partial B_{\rho}\left(y^{(k)}\right)}\left\{\frac{\partial}{\partial y_{1}^{(j)}} \log \left|x-y^{(j)}\right| \frac{\partial}{\partial \nu_{x}} S\left(x, y^{(j)}\right)+\frac{\partial}{\partial y_{1}^{(j)}} S\left(x, y^{(j)}\right) \frac{\partial}{\partial \nu_{x}} G\left(x, y^{(j)}\right)\right\} d \sigma_{x} \\
& +\sum_{k=1}^{m} \sum_{\ell \neq j} \int_{\partial B_{\rho}\left(y^{(k)}\right)} d_{j} d_{\ell} \frac{\partial}{\partial y_{1}^{(j)}} G\left(x, y^{(j)}\right) \cdot \nabla_{x} G\left(x, y^{(\ell)}\right) d x
\end{aligned}
$$




$$
-2 \pi \frac{\partial}{\partial y_{1}^{(j)}} S\left(y^{(j)}, y^{(j)}\right)-2 \pi \sum_{k \neq j} d_{j} d_{k} \frac{\partial}{\partial y_{1}^{(j)}} G\left(y^{(k)}, y^{(j)}\right)+O(\rho)
$$

Since

$$
\frac{\partial}{\partial y_{1}^{(j)}} V(\boldsymbol{y})=-2 \pi \frac{\partial}{\partial y_{1}^{(j)}} S\left(y^{(j)}, y^{(j)}\right)-2 \pi \sum_{k \neq j} d_{j} d_{k} \frac{\partial}{\partial y_{1}^{(j)}} G\left(y^{(k)}, y^{(j)}\right),
$$

adding (5.14) to (5.17), we obtain the desired result (5.9).

6. Dynamics for the vortex solution. Given small $\rho_{0}>0$, we let

$$
Y_{0}:=\left\{\boldsymbol{y} \in \hat{\Omega}^{m}:\left|y^{(j)}-y^{(k)}\right|>\frac{5}{2} \rho_{0}, \quad \operatorname{dist}\left(y^{(j)}, \partial \Omega\right)>2 \rho_{0}\right\} \subset Y
$$

( $Y$ is defined by (4.1)) and

$$
\mathcal{M}_{\epsilon}:=\left\{u=u_{\epsilon}(\cdot, \boldsymbol{y}): \boldsymbol{y} \in Y_{0}\right\}
$$

We denote a Banach space of continuous functions by

$$
X:=C^{0}(\bar{\Omega} ; \mathbb{C}) \quad \text { with norm } \quad\|u\|_{0}:=\sup _{x \in \bar{\Omega}}|u(x)|
$$

Then $\mathcal{M}_{\epsilon}$ is a $2 m$-dimensional submanifold of $X$. We discuss the dynamics of the solution to (1.1) in a neighborhood of the manifold $\mathcal{M}_{\epsilon}$. We derive the motion law projected on $\mathcal{M}_{\epsilon}$. To carry out it, we borrow the idea from $\S 2$ of [7]. We, however, need a more careful consideration because our approximate solution is not so nice as in [7], where their approximate solution has exponentially small error.

Define an operator $K_{\epsilon}: \overline{Y_{0}} \times X \longmapsto \mathbb{R}^{2 m}$ by

$$
\begin{aligned}
K_{\epsilon}(\boldsymbol{y}, u) & =\left(K_{1}^{(1)}, K_{2}^{(1)}, \cdots, K_{1}^{(j)}, K_{2}^{(j)}, \cdots, K_{1}^{(m)}, K_{2}^{(m)}\right) \\
K_{q}^{(j)} & =K_{q}^{(j)}(\boldsymbol{y}, u):=\left\langle u-u_{\epsilon}(\cdot, \boldsymbol{y}), \frac{\partial u_{\epsilon}}{\partial y_{q}^{(j)}}(\cdot, \boldsymbol{y})\right\rangle_{L^{2}}, \quad q=1,2 .
\end{aligned}
$$

Note that

$$
K_{\epsilon}\left(\boldsymbol{y}, u_{\epsilon}(\cdot, \boldsymbol{y})\right)=0
$$

We first show

Lemma 6.1. $K_{\epsilon}(\boldsymbol{y}, u)$ is a $C^{1}$ function in $(\boldsymbol{y}, u)$ and the derivative with respect to $\boldsymbol{y}$ at $(\boldsymbol{y}, u)=\left(\boldsymbol{y}, u_{\epsilon}(\cdot ; \boldsymbol{y})\right)$ can be written as

$$
\frac{\partial}{\partial \boldsymbol{y}} K_{\epsilon}\left(\boldsymbol{y}, u_{\epsilon}(\cdot ; \boldsymbol{y})\right)=-\pi \log (1 / \epsilon) I_{\epsilon}, \quad I_{\epsilon}=I_{2 m}+O(1 / \log (1 / \epsilon))
$$

where $I_{2 m}$ is the $2 m \times 2 m$-identity matrix. Moreover for any $\boldsymbol{y}_{0} \in \overline{Y_{0}}$,

$$
\left|\frac{\partial}{\partial \boldsymbol{y}} K_{\epsilon}(\boldsymbol{y}, u)-\frac{\partial}{\partial \boldsymbol{y}} K_{\epsilon}\left(\boldsymbol{y}_{0}, u_{\epsilon}\left(\cdot, \boldsymbol{y}_{0}\right)\right)\right| \leq C_{1}\left\{1+\log (1 / \epsilon)\left\|u-u_{\epsilon}(\cdot, \boldsymbol{y})\right\|_{0}\right\}
$$

holds. 
Proof. It is easy to see that $K_{\epsilon}(\boldsymbol{y}, u)$ is $C^{1}$. Since

$$
\frac{\partial K_{q}^{(j)}}{\partial y_{p}^{(k)}}\left(\boldsymbol{y}, u_{\epsilon}(\cdot ; \boldsymbol{y})\right)=-\left\langle\frac{\partial u_{\epsilon}}{\partial y_{p}^{(k)}}(\cdot, \boldsymbol{y}), \frac{\partial u_{\epsilon}}{\partial y_{q}^{(j)}}(\cdot, \boldsymbol{y})\right\rangle_{L^{2}},
$$

(6.5) immediately follows from (4.24) of Lemma 4.3. To prove (6.6), we use (4.25) in addition to (4.24). In fact

$$
\frac{\partial K_{q}^{(j)}}{\partial y_{p}^{(k)}}(\boldsymbol{y}, u)=-\left\langle\frac{\partial u_{\epsilon}}{\partial y_{p}^{(k)}}(\cdot, \boldsymbol{y}), \frac{\partial u_{\epsilon}}{\partial y_{q}^{(j)}}(\cdot, \boldsymbol{y})\right\rangle_{L^{2}}+\left\langle u-u_{\epsilon}(\cdot, \boldsymbol{y}), \frac{\partial^{2} u_{\epsilon}}{\partial y_{p}^{(k)} \partial y_{q}^{(j)}}(\cdot, \boldsymbol{y})\right\rangle_{L^{2}} .
$$

Noticing that (4.24) holds for $\boldsymbol{y}$ and $\boldsymbol{y}_{0}$, we easily get to (6.6) by (4.24) and (4.25).

Next for arbitrarily given $\boldsymbol{y}_{0} \in \overline{Y_{0}}$, we define

$$
\mathcal{H}_{\epsilon}(\boldsymbol{y}, u):=\boldsymbol{y}-A_{\epsilon}\left(\boldsymbol{y}_{0}\right)^{-1} K_{\epsilon}(\boldsymbol{y}, u), \quad A_{\epsilon}\left(\boldsymbol{y}_{0}\right):=\frac{\partial}{\partial \boldsymbol{y}} K_{\epsilon}\left(\boldsymbol{y}_{0}, u_{\epsilon}\left(\cdot, \boldsymbol{y}_{0}\right)\right)
$$

Put

(6.8) $V_{\eta}\left(\boldsymbol{y}_{0}\right):=\left\{\boldsymbol{y} \in Y:\left|\boldsymbol{y}-\boldsymbol{y}_{0}\right| \leq \eta\right\}, \quad X_{\delta}\left(\boldsymbol{y}_{0}\right):=\left\{u \in X:\left\|u-u_{\epsilon}\left(\cdot, \boldsymbol{y}_{0}\right)\right\|_{0} \leq \delta\right\}$.

Then $\mathcal{H}_{\epsilon}: V_{\eta}\left(\boldsymbol{y}_{0}\right) \times X_{\delta}\left(\boldsymbol{y}_{0}\right) \rightarrow \mathbb{R}^{2 m}$ and

$$
\mathcal{H}_{\epsilon}(\boldsymbol{y}, u)=\boldsymbol{y} \Leftrightarrow K_{\epsilon}(\boldsymbol{y}, u)=0, \quad \mathcal{H}_{\epsilon}\left(\boldsymbol{y}_{0}, u_{\epsilon}\left(\cdot, \boldsymbol{y}_{0}\right)\right)=\boldsymbol{y}_{0}
$$

hold. We prove that $\mathcal{H}_{\epsilon}$ is a contraction mapping.

LEMMA 6.2. There exist small $\delta_{1}>0$ and $\epsilon_{1}>0$ such that if $\delta \in\left(0, \delta_{1}\right)$ and $\epsilon \in\left(0, \epsilon_{1}\right)$, for any $\boldsymbol{y}_{0} \in \overline{Y_{0}}$ there exists an $\eta=\eta\left(\boldsymbol{y}_{0}, \epsilon\right)>0$ for which the map $\mathcal{H}_{\epsilon}$ of (6.7) carries $V_{\eta}\left(\boldsymbol{y}_{0}\right) \times X_{\delta}\left(\boldsymbol{y}_{0}\right)$ into $V_{\eta}\left(\boldsymbol{y}_{0}\right)$ and

$$
\left|\mathcal{H}_{\epsilon}\left(\boldsymbol{y}_{1}, u\right)-\mathcal{H}_{\epsilon}\left(\boldsymbol{y}_{2}, u\right)\right| \leq \frac{1}{2}\left|\boldsymbol{y}_{1}-\boldsymbol{y}_{2}\right|, \quad \boldsymbol{y}_{j} \in V_{\eta}\left(\boldsymbol{y}_{0}\right) \quad(j=1,2)
$$

Proof. By the definition of $\mathcal{H}_{\epsilon}$ we can compute

$$
\begin{aligned}
& \left|\mathcal{H}_{\epsilon}\left(\boldsymbol{y}_{1}, u\right)-\mathcal{H}_{\epsilon}\left(\boldsymbol{y}_{2}, u\right)\right| \\
= & \left|A_{\epsilon}\left(\boldsymbol{y}_{0}\right)^{-1}\left\{A_{\epsilon}\left(\boldsymbol{y}_{0}\right)\left(\boldsymbol{y}_{1}-\boldsymbol{y}_{2}\right)-\left(K_{\epsilon}\left(\boldsymbol{y}_{1}, u\right)-K_{\epsilon}\left(\boldsymbol{y}_{2}, u\right)\right)\right\}\right| \\
\leq & \frac{\left|I_{\epsilon}^{-1}\right|_{o p}}{\pi \log (1 / \epsilon)}\left|A_{\epsilon}\left(\boldsymbol{y}_{0}\right)-\int_{0}^{1} \frac{\partial}{\partial \boldsymbol{y}} K\left(\boldsymbol{y}_{2}+t\left(\boldsymbol{y}_{1}-\boldsymbol{y}_{2}\right), u\right) d t\right|_{o p}\left|\boldsymbol{y}_{1}-\boldsymbol{y}_{2}\right|
\end{aligned}
$$

where $|\cdot|_{o p}$ denotes the operater norm for linear maps from $\mathbb{R}^{2 m}$ into itself. We estimate

$$
\begin{aligned}
& \left|A_{\epsilon}\left(\boldsymbol{y}_{0}\right)-\int_{0}^{1} \frac{\partial}{\partial y} K\left(\boldsymbol{y}_{2}+t\left(\boldsymbol{y}_{1}-\boldsymbol{y}_{2}\right), u\right) d t\right|_{o p} \\
= & \int_{0}^{1}\left|\frac{\partial}{\partial \boldsymbol{y}} K\left(\boldsymbol{y}_{0}, u_{\epsilon}\left(\cdot, \boldsymbol{y}_{0}\right)\right)-\frac{\partial}{\partial \boldsymbol{y}} K\left(\boldsymbol{y}_{2}+t\left(\boldsymbol{y}_{1}-\boldsymbol{y}_{2}\right), u\right)\right|_{o p} d t \\
\leq & C_{1}\left\{1+\left(\log \frac{1}{\epsilon}\right)\left\|u-u_{\epsilon}(\cdot, \tilde{\boldsymbol{y}})\right\|_{0}\right\} \\
\leq & C_{1}\left[1+\left(\log \frac{1}{\epsilon}\right)\left\{\left\|u-u_{\epsilon}\left(\cdot, \boldsymbol{y}_{0}\right)\right\|_{0}+\left\|u_{\epsilon}\left(\cdot, \boldsymbol{y}_{0}\right)-u_{\epsilon}(\cdot, \tilde{\boldsymbol{y}})\right\|_{0}\right\}\right] .
\end{aligned}
$$


where we put $\tilde{\boldsymbol{y}}=\boldsymbol{y}_{2}+t\left(\boldsymbol{y}_{1}-\boldsymbol{y}_{2}\right)$ and used (6.6) of Lemma 6.1. Let $\delta_{1}>0$ be a number satisfying $\delta_{1}<\pi / 6$ and take $\eta=\eta\left(\boldsymbol{y}_{0}, \epsilon\right)$ so that

$$
\left\|u_{\epsilon}\left(\cdot, \boldsymbol{y}_{0}\right)-u_{\epsilon}(\cdot, \boldsymbol{y})\right\|_{0} \leq \frac{\pi}{6}
$$

holds for $\left|\boldsymbol{y}-\boldsymbol{y}_{0}\right|<\eta\left(\boldsymbol{y}_{0}, \epsilon\right)$. Then inserting (6.11) into (6.10) yields (6.9).

To prove

$$
\mathcal{H}_{\epsilon}: V_{\eta}\left(\boldsymbol{y}_{0}\right) \times X_{\delta}\left(\boldsymbol{y}_{0}\right) \longmapsto V_{\eta}\left(\boldsymbol{y}_{0}\right)
$$

we notice

$$
\left|\mathcal{H}_{\epsilon}(\boldsymbol{y}, u)-\boldsymbol{y}_{0}\right|=\left|\mathcal{H}_{\epsilon}(\boldsymbol{y}, u)-\mathcal{H}_{\epsilon}\left(\boldsymbol{y}_{0}, u_{\epsilon}\left(\cdot ; \boldsymbol{y}_{0}\right)\right)\right| \leq \frac{1}{2}\left|\boldsymbol{y}-\boldsymbol{y}_{0}\right| \leq \frac{1}{2} \eta
$$

We concluded the proof.

By the above lemma, we can use the uniform contraction mapping principle (see Chap.1, 1.2.6 of [11]) to obtain the following lemma:

Lemma 6.3. For each $u \in X_{\delta}\left(\boldsymbol{y}_{0}\right)$ there is a fixed point of $\mathcal{H}_{\epsilon}, \boldsymbol{y}=\boldsymbol{y}_{\epsilon}(u) \in V_{\eta}\left(\boldsymbol{y}_{0}\right)$ such that $\boldsymbol{y}_{\epsilon}(u)$ is a $C^{1}$ function satisfying $\boldsymbol{y}_{\epsilon}\left(u_{\epsilon}\left(\boldsymbol{y}_{0}\right)\right)=\boldsymbol{y}_{0}$, where $\eta\left(=\eta\left(\boldsymbol{y}_{0}, \epsilon\right)\right)$ is as in Lemma 6.2.

We can extend the function $\boldsymbol{y}_{\epsilon}(u)$ to the one defined in a neighborhood of $\mathcal{M}_{\epsilon}$.

Lemma 6.4. Let $Y$ be as in (4.1) and $\delta_{1}$ be a number as in Lemma 6.2. For any $\delta \in\left(0, \delta_{1}\right)$, define

$$
\mathcal{U}_{\delta}\left(\mathcal{M}_{\epsilon}\right):=\left\{u \in X: \min _{\boldsymbol{y} \in \overline{Y_{0}}}\left\|u-u_{\epsilon}(\cdot ; \boldsymbol{y})\right\|_{0}<\delta\right\}
$$

where $\mathcal{M}_{\epsilon}$ is defined by (6.2). Then there is a $C^{1}$ mapping $\boldsymbol{y}_{\epsilon}: \mathcal{U}_{\delta}\left(\mathcal{M}_{\epsilon}\right) \rightarrow Y$ such that $K_{\epsilon}\left(\boldsymbol{y}_{\epsilon}(u), u\right)=0$. Moreover for $u \in \mathcal{U}_{\delta}\left(\mathcal{M}_{\epsilon}\right), \boldsymbol{y}_{\epsilon}(u)$ minimizes $\left\|u-u_{\epsilon}(\cdot ; \boldsymbol{y})\right\|_{L^{2}(\Omega)}$.

Proof. By virtue of the compactness of $\overline{Y_{0}}$ and that $\delta_{1}$ can be taken uniformly for $\boldsymbol{y}_{0} \in \overline{Y_{0}}$, the former assertion of the lemma immediately follows. To prove the latter part, put

$$
\alpha(\boldsymbol{\xi}):=\left\|u-u_{\epsilon}\left(\cdot ; \boldsymbol{y}_{\epsilon}(u)+\boldsymbol{\xi}\right)\right\|_{L^{2}(\Omega)}^{2}
$$

Then one can easily verify

$$
\frac{\partial}{\partial \boldsymbol{\xi}} \alpha(0)=K_{\epsilon}\left(\boldsymbol{y}_{\epsilon}(u), u\right)=0
$$

and

$$
\begin{aligned}
\frac{\partial^{2}}{\partial \xi_{p}^{(k)} \partial \xi_{q}^{(j)}} \alpha(0)= & -\left\langle\frac{\partial u_{\epsilon}}{\partial y_{p}^{(k)}}\left(\cdot, \boldsymbol{y}_{\epsilon}(u)\right), \frac{\partial u_{\epsilon}}{\partial y_{q}^{(j)}}\left(\cdot, \boldsymbol{y}_{\epsilon}(u)\right)\right\rangle_{L^{2}} \\
& +\left\langle u-u_{\epsilon}\left(\cdot, \boldsymbol{y}_{\epsilon}(u)\right), \frac{\partial^{2} u_{\epsilon}}{\partial y_{p}^{(k)} \partial y_{q}^{(j)}}\left(\cdot, \boldsymbol{y}_{\epsilon}(u)\right)\right\rangle_{L^{2}}
\end{aligned}
$$


By Lemma 4.3 there is a small $\delta_{1}>0$ such that if $\delta \in\left(0, \delta_{1}\right)$, every principal curvature of $\alpha(\xi)$ at $\boldsymbol{\xi}=0$ is positive for any small $\epsilon>0$. This proved the lemma.

We have the main result on the dynamics of vortex solutions.

THEOREM 6.5. Assume that $\rho(\epsilon)$ is a positive function satisfying

$$
\lim _{\epsilon \rightarrow+0} \rho(\epsilon)=0, \quad \lim _{\epsilon \rightarrow+0} \frac{\epsilon}{\rho(\epsilon)^{3 / 2}}=0,
$$

and that $u_{\epsilon}(x ; \boldsymbol{y}), \boldsymbol{y} \in Y$ are the functions defined by (4.7), where $Y$ is as in (4.1). Let $\boldsymbol{y}_{\epsilon}: \mathcal{U}_{\delta}\left(\mathcal{M}_{\epsilon}\right) \rightarrow Y$ be a $C^{1}$ function appearing in Lemma 6.4. If for a positive $T>0$, a solution $u^{\epsilon}(x, t)$ of $(1.1)$ in $\mathcal{U}_{\delta}\left(\mathcal{M}_{\epsilon}\right)$ satisfies

$$
\left\|u^{\epsilon}(\cdot, t)-u_{\epsilon}\left(\cdot ; \boldsymbol{y}_{\epsilon}\left(u^{\epsilon}(\cdot, t)\right)\right)\right\|_{0}=o(\epsilon), \quad t \in[0, T]
$$

then there is a $\epsilon_{1}>0$ such that $\boldsymbol{y}=\boldsymbol{y}_{\epsilon}\left(u^{\epsilon}(\cdot, t)\right)$ admits

$$
\log (1 / \epsilon) \frac{d}{d t} \boldsymbol{y}=-\frac{1}{\pi} \nabla V(\boldsymbol{y})+o(1) .
$$

Proof. For simplicity of notation, we drop $\epsilon$ in the solution $u^{\epsilon}(x, t)$ and write $\boldsymbol{y}(t)=\boldsymbol{y}_{\epsilon}(u(\cdot, t))$. We also simply write $\langle\cdot, \cdot\rangle$ for $\langle\cdot, \cdot\rangle_{L^{2}}$. Lemma 6.4 tells that

$$
\mathcal{K}_{\epsilon}(\boldsymbol{y}(t), u(\cdot, t))=0
$$

which implies

$$
\left\langle u(\cdot, t)-u_{\epsilon}(\cdot ; \boldsymbol{y}(t)), \frac{\partial}{\partial y_{q}^{(j)}} u_{\epsilon}(\cdot ; \boldsymbol{y}(t))\right\rangle=0, \quad q=1,2, \quad 1 \leq j \leq m
$$

Differentiating (6.16) with respect to $t$ yields

$$
\begin{aligned}
& \left\langle u_{t}-\sum_{p, k} \frac{\partial u_{\epsilon}}{\partial y_{p}^{(k)}} \dot{y}_{p}^{(k)}, \frac{\partial u_{\epsilon}}{\partial y_{q}^{(j)}}\right\rangle+\left\langle u(\cdot, t)-u_{\epsilon}(\cdot ; \boldsymbol{y}(t)), \sum_{p, k} \frac{\partial^{2} u_{\epsilon}}{\partial y_{p}^{(k)} \partial y_{q}^{(j)}} \dot{y}_{p}^{(k)}\right\rangle \\
= & \left\langle\mathcal{F}(u(\cdot, t)), \frac{\partial u_{\epsilon}}{\partial y_{q}^{(j)}}\right\rangle \\
& -\sum_{p, k}\left\{\left\langle\frac{\partial u_{\epsilon}}{\partial y_{p}^{(k)}}, \frac{\partial u_{\epsilon}}{\partial y_{q}^{(j)}}\right\rangle-\left\langle u(\cdot, t)-u_{\epsilon}(\cdot ; \boldsymbol{y}(t)), \frac{\partial^{2} u_{\epsilon}}{\partial y_{p}^{(k)} \partial y_{q}^{(j)}}\right\rangle\right\} \dot{y}_{p}^{(k)}=0,
\end{aligned}
$$

where $\dot{y}=d y / d t$. By putting

$$
v_{\epsilon}(x, t):=u(x, t)-u_{\epsilon}(x ; \boldsymbol{y}(t))
$$

we obtain

$$
\begin{aligned}
& \sum_{p, k}\left\{\left\langle\frac{\partial u_{\epsilon}}{\partial y_{p}^{(k)}}, \frac{\partial u_{\epsilon}}{\partial y_{q}^{(j)}}\right\rangle-\left\langle v_{\epsilon}(\cdot, t), \frac{\partial^{2} u_{\epsilon}}{\partial y_{p}^{(k)} \partial y_{q}^{(j)}}\right\rangle\right\} \dot{y}_{p}^{(k)} \\
= & \left\langle\mathcal{F}\left(u_{\epsilon}+v_{\epsilon}(\cdot, t)\right), \frac{\partial u_{\epsilon}}{\partial y_{q}^{(j)}}\right\rangle
\end{aligned}
$$


$\left(u_{\epsilon}=u_{\epsilon}(\cdot ; \boldsymbol{y}(t))\right)$. We write

$$
\begin{aligned}
\mathcal{F}\left(u_{\epsilon}+v_{\epsilon}\right) & =\mathcal{F}\left(u_{\epsilon}\right)+\mathcal{L}\left(v_{\epsilon}\right)+N\left(v_{\epsilon}\right) \\
\mathcal{L}\left(v_{\epsilon}\right) & :=\Delta v_{\epsilon}+\frac{1}{\epsilon^{2}}\left(1-\left|u_{\epsilon}\right|^{2}\right) v_{\epsilon}-\frac{2}{\epsilon^{2}} \operatorname{Re}\left(u_{\epsilon} \overline{v_{\epsilon}}\right) u_{\epsilon}, \\
N\left(v_{\epsilon}\right) & :=\frac{1}{\epsilon^{2}}\left\{\left|v_{\epsilon}\right|^{2} u_{\epsilon}+2 \operatorname{Re}\left(u_{\epsilon} \overline{v_{\epsilon}}\right) v_{\epsilon}+\left|v_{\epsilon}\right|^{2} v_{\epsilon}\right\} .
\end{aligned}
$$

We can easily verify by integration by part that

$$
\begin{aligned}
\left\langle\mathcal{F}\left(u_{\epsilon}\right), \frac{\partial u_{\epsilon}}{\partial y_{q}^{(j)}}\right\rangle & =-\frac{1}{2} \int_{\Omega} \frac{\partial}{\partial y_{q}^{(j)}}\left\{\left|\nabla u_{\epsilon}\right|^{2}+\frac{1}{2}\left(1-\left|u_{\epsilon}\right|^{2}\right)^{2}\right\} d x+O\left(\epsilon^{2}\right) \\
& =-\frac{\partial}{\partial y_{q}^{(j)}} E_{\epsilon}\left(u_{\epsilon}\right)+O\left(\epsilon^{2}\right) \\
\left\langle\mathcal{L}\left(v_{\epsilon}\right), \frac{\partial u_{\epsilon}}{\partial y_{q}^{(j)}}\right\rangle & =\int_{\partial \Omega} \frac{\partial v_{\epsilon}}{\partial \nu} \frac{\partial u_{\epsilon}}{\partial y_{q}^{(j)}} d \sigma-\int_{\partial \Omega} v_{\epsilon} \frac{\partial}{\partial \nu} \frac{\partial u_{\epsilon}}{\partial y_{q}^{(j)}} d \sigma+\left\langle v_{\epsilon}, \mathcal{L}\left(\partial u_{\epsilon} / \partial y_{q}^{(j)}\right)\right\rangle
\end{aligned}
$$

Recall

$$
\frac{\partial v_{\epsilon}}{\partial \nu}=-\frac{\partial u_{\epsilon}}{\partial \nu}=O\left(\epsilon^{2}\right), \quad x \in \partial \Omega .
$$

Moreover applying Lemmas 4.2 and 5.1, we obtain

$$
\left|\left\langle v_{\epsilon}, \mathcal{L}\left(\partial u_{\epsilon} / \partial y_{q}^{(j)}\right)\right\rangle\right|=\left|\left\langle v_{\epsilon}, \frac{\partial}{\partial y_{q}^{(j)}} \mathcal{F}\left(u_{\epsilon}\right)\right\rangle\right| \leq C_{1} \max \left\{\log (1 / \epsilon), \epsilon^{2} / \rho^{2}\right\}\left\|v_{\epsilon}\right\|_{0},
$$

and

$$
\left|\left\langle N\left(v_{\epsilon}\right), \frac{\partial u_{\epsilon}}{\partial y_{q}^{(j)}}\right\rangle\right| \leq C_{1} \frac{1}{\epsilon^{2}}\left\|v_{\epsilon}\right\|_{0}^{2} .
$$

Hence from the assumption (6.14)

$$
\left\langle\mathcal{F}\left(u_{\epsilon}+v_{\epsilon}\right), \frac{\partial u_{\epsilon}}{\partial y_{q}^{(j)}}\right\rangle=-\frac{\partial}{\partial y_{q}^{(j)}} E_{\epsilon}\left(u_{\epsilon}\right)+o(1)
$$

follows.

We apply Lemma 4.3 to the left hand side of (6.17) and use (5.2) of Lemma 5.1 in (6.20) to obtain

$$
\log (1 / \epsilon) \dot{y}_{q}^{(j)}=-\frac{1}{\pi} \frac{\partial}{\partial y_{q}^{(j)}} V(\boldsymbol{y})+o(1),
$$

which is the desired equation. 
Acknowledgement. The authors would like to express their thanks to Professor H. Ninomiya for useful comments. The second author was supported in part by the Ryukoku Joint Research Center for Science and Technology.

\section{REFERENCES}

[1] G. Bellettini AND G. Fusco, Stable dynamics of spikes in solutions to a system of activatorinhibitor type, preprint.

[2] F. Bethuel, H. Brezis And F. HÉLein, Ginzburg-Landau Vortices, Birkhäuser, 1994.

[3] J. Carr And R.L. Pego, Metastable patterns in solutions of $u_{t}=\epsilon^{2} u_{x x}-f(u)$, Comm. Pure Appl. Math., 42 (1989), pp. 523-576.

[4] X. CHEN, Generation and propagation of interfaces for reaction-diffusion equations, J. Differential Equations, 96 (1992), pp. 116-141.

[5] X. Chen, C.M. ElliotT AND T. QI, Shooting method for vortex solutions of a complex-valued Ginzburg-Landau equation, Proc. Roy. Soc. Edingburgh Sect. A, 124 (1994), pp. 1075-1088.

[6] W. E, Dynamics of vortices in Ginzburg-Landau theories with applications to superconductivity, Phys. D, 77 (1994), pp. 383-404.

[7] J.-P. Eckmann and J. Rougenmont, Coarsening by Ginzburg-Landau dynamics, Comm. Math. Phys. 199 (1998), pp. 441-470.

[8] S. -I. EI AND E. YANAGIDA, Slow dynamics of interfaces in the Allen-Cahn equation on a strip-like domain, SIAM J. Math. Anal., 29 (1998), pp. 555-595.

[9] S. -I. EI AND J. WEI, Dynamics of interior spike solutions, preprint.

[10] P. FIFE, Dynamics of Internal Layers and Diffusive Interfaces, CBMS-NSF, Regional Conference Series in Applied Mathematics, 53, SIAM, Philadelphia (1988).

[11] D. Henry, Geometric Theory of Semilinear Parabolic Equations, Springer-Verlag, New York, 1981.

[12] R. L. Jerrard And H. M. Soner, Dynamics of Ginzburg-Landau Vortices, Arch. Ration. Mech. Anal. 142 (1998), pp. 99-125.

[13] S. JIMBO AND Y. MORITA, Stability of non-constant steady state solutions to a Ginzburg-Landau equation in higher space dimensions, Nonlinear Anal., 22 (1994), pp. 753-770.

[14] S. JIMBO AND Y. MorITA, Stable solutions with zeros to the Ginzburg-Landau equation with Neumann boundary condition, J. Differential Equations, 128 (1996), pp. 596-613.

[15] S. JIMBO AND Y. MORITA, Stable vortex solutions to the Ginzburg-Landau equation with a variable coefficient in a disk, J. Differential Equations, 155 (1999), pp. 153-176.

[16] S. JIMBO AND Y. MORITA, Notes on the limit equation of vortex motion for the GinzburgLandau equation with Neumann condition, Japan J. Indust. Appl. Math., 18 (2001), pp. $483-501$.

[17] S. JIMBO, Y. MORITA AND J. ZHAI, Ginzburg-Landau equation and stable steady state solutions in a non-trivial domain, Comm. Partial Differential Equations, 20 (1995), pp. 93-2112.

[18] Y. Kametaka, On a Nonlinear Bessel Equation, Pub. RIMS, Kyoto Univ. 8 (1972), pp. 151200.

[19] F. H. Lin, Some Dynamical properties of Ginzburg-Landau vortices, Comm. Pure Appl. Math. 49 (1996), pp. 323-359.

[20] F. H. LiN, A remark on the previous paper "Some dynamical properties of Ginzburg-Landau vortices, Comm. Pure Appl. Math. 49 (1996), pp. 361-364.

[21] F. H. LiN, Complex Ginzburg-Landau equations and dyanamics of vortices, filaments, and codimension-2 submanifolds, Comm. Pure Appl. Math. 51 (1998), pp. 385-441.

[22] F. H. Lin AND J. X. XIN, On the dynamical law of the Ginzburg-Landau vortices on the plane, Comm. Pure and Appl. Math. 52 (1999), pp. 1189-1212.

[23] T. C. LIN, Spectrum of the linearized operator for the Ginzburg-Landau equation, Electron. J. Diffrential Equations, 42 (2000), pp. 25.

[24] P. MIRONESCU, On the stability of radial solutions of the Ginzburg-Landau equation, J. of Funct. Anal., 130 (1995), pp. 334-344.

[25] J. NeU, Vortices in complex scalar fields, Phys. D, 43 (1990), pp. 385-406.

[26] J. Rubinstein And P. Sternberg, On the slow motion of vortices in the Ginzburg-Landau heat flow, SIAM J. Math. Anal., 26 (1995), pp. 1452-1466.

[27] J. WEI AND M. WINTER, On multiple spike solutions for the two-dimensional Gierer-Meinhardt system; the strong coupling case, preprint. 
S. JIMBO AND Y. MORITA 